\title{
Time-resolved expression analysis comparing two selective retinal cell ablation paradigms in zebrafish reveals shared and cell-specific regenerative regulatory networks.
}

Steven L. Walker ${ }^{1,2 *}$, Guohua Wang ${ }^{3,4 *}$, Kevin B. Emmerich ${ }^{3,5}$, Fang Wang ${ }^{3}$, David T. White ${ }^{1,3}$, Yong Teng ${ }^{6}$, Meera T. Saxena ${ }^{3}$, Jiang Qian ${ }^{3 \#}$, and Jeff S. Mumm ${ }^{1,3,5,7,8 \#}$

*Co-first authors

${ }^{\#}$ Co-corresponding authors: jmumm3@jhmi.edu, jiang.qian@jhmi.edu

1. Department of Cellular Biology and Anatomy, Medical College of Georgia, Augusta University, Augusta, Georgia, United States of America

2. Current address: School of Biomedical Sciences, The Chinese University of Hong Kong, N.T., Hong Kong

3. Department of Ophthalmology, Wilmer Eye Institute, Johns Hopkins University, Baltimore, MD, USA

4. Current address: School of Computer Science and Technology of Harbin Institute of Technology, Harbin, China

5. McKusick-Nathans Institute of the Department of Genetic Medicine, Johns Hopkins School of Medicine, Baltimore, MD, USA

6. Georgia Cancer Center, Augusta University, Augusta, Georgia, United States of America

7. Department of Neuroscience, Johns Hopkins University, Baltimore, MD, USA

8. Center for Nanomedicine, Johns Hopkins University, Baltimore, MD, USA 


\begin{abstract}
Zebrafish are an effective model organism for retinal regeneration studies. Regenerated retinal cells are derived from Müller glia (MG) stem cells. Mammalian MG can also produce new retinal neurons; however, this regenerative potential remains dormant in the absence of genetic and/or chemical stimulation. An understanding of how the regenerative potential of MG is regulated could aid efforts to promote regeneration therapeutically. Following widespread retinal cell death, developmental signaling coordinates regeneration. Less is known about how MG respond to the loss of specific cell types, i.e., paradigms similar to retinal degenerative diseases. To address this, transcriptomic responses to the selective loss of rod photoreceptors or retinal bipolar cells were compared over twelve timepoints spanning cell degeneration and regeneration. Shared and paradigm-specific expression changes were identified throughout regeneration. Overall, paradigm-specific changes predominated, suggesting cell-specific mechanisms for activating MG stem cells. One particularly interested finding new for retinal regeneration was early regulation of SOCS family genes. These are associated with stat 3 activation and the JAK/STAT pathway which has been well documented in similar studies and was further supported in our analyses. These data support the concept that selective retinal cell loss can elicit cell-specific regenerative programs and provide novel insights into retinal regeneration.
\end{abstract}

\title{
Introduction
}

Specific neuronal subtypes are lost at the onset of most retinal degenerative diseases. Regenerative therapeutic approaches for replacing lost retinal cells have the potential to restore visual function. However, our understanding of how the retina responds to selective neuronal cell loss, or how individual cell types can be regenerated, is nascent. Regenerative biology has been predominated by large-scale injury paradigms that incur damage across multiple cell types (e.g., tissue regeneration) (1) or tissues (e.g., limb replacement) (2). These paradigms elicit classic wound healing responses and trigger regenerative processes that recapitulate developmental and/or homeostatic processes (3). Mechanisms regulating retinal regeneration following non-specific cell loss in zebrafish also largely involve developmental pathways (4-7). In contrast, the responsiveness and regenerative properties of stem cells following the discrete loss of retinal cell subtypes remain less well undefined. Intriguingly, recent studies suggest that selective retinal cell loss in zebrafish elicits a fate-biased regenerative response; i.e., selective cell replacement (8-11). To increase understanding of fate-biased regenerative responses at the transcriptomic level, we used time-resolved gene expression profiling to compare two cell-specific ablation paradigms in the zebrafish retina involving the targeted loss of 1) bipolar cells, versus, 2) rod photoreceptors.

Retinal regeneration research has largely involved three main non-specific injury paradigms: lightinduced ablation $(12-15)$, toxin-induced ablation $(16,17)$ and mechanical wounding $(18,19)$. Initial mechanical wounding studies involved surgical excisions of retinal quadrants with classic epimorphic regeneration hallmarks: rapid wound closure and the formation of blastema-like zones of proliferative cells (18). Other paradigms eliminate discrete somal layers of the retina. Light ablation approaches eliminate rod and cone photoreceptors (12-15), while neurotoxins ablate interneurons in the inner nuclear layer (INL) and retinal ganglion cells (RGCs) (17). Collectively, these studies implicated two inducible stem cell niches in the teleost retina; outer nuclear layer (ONL) progenitor cells thought to be committed to producing only rod cells $(20,21)$, and an INL- 
localized retinal stem cell $(22,23)$. Multiple labs have demonstrated that Müller glia (MG) function as the multipotent INL stem cell in zebrafish, capable of regenerating all lost retinal cell types (47). Moreover, mammalian MG have recently been shown retain the capacity to function as retinal stem cells (24), highlighting the need to understand how MG regenerative potential is regulated. Similar to large-scale injury paradigms across regenerative biology, developmental pathways govern retinal regeneration following non-specific cell loss (4-7).

Most retinal degenerative diseases are linked to the loss of discrete cell types. Therapies aimed at stimulating the reparative capacities of human $\mathrm{MG}$ therefore requires increased understanding of the signaling pathways controlling MG responses to selective retinal cell loss. We adapted an inducible targeted cell ablation method (25-29) to zebrafish (30-32) to enable a broad range of cell-specific regeneration paradigms (28). Cell-specific transgenic expression of a prodrug converting bacterial enzyme nitroreductase (NTR) renders cells susceptible to prodrugs, such as metronidazole (MTZ), resulting in DNA-damage induced selective cell death $(33,34)$. Coexpression of a fluorescent reporter in NTR-targeted cells allows the regenerative process to be characterized in detail using in vivo time-lapse imaging $(32,35)$ or quantified using highthroughput methods $(36,37)$. Using this approach, recent studies have shown that selective retinal cell loss does not trigger a developmental response but rather a fate-biased regenerative process (8-11). These data suggest differential regulation of selective and non-selective cellular regeneration. Using a selective rod cell ablation model, Hyde and colleagues have shown that the extent of cell loss is an additional factor impacting the responsiveness of retinal stem cell niches $(33,62)$ : ONL progenitors being solely responsive to limited rod cell loss while widespread loss elicited both MG and ONL progenitor responses.

One of the main advantages of an inducible targeted cell ablation system is the ability to synchronize the onset of cell death. This facilitates temporally-resolved characterization of the cellular and molecular events mediating degenerative and regenerative processes-i.e. activation, dedifferentiation, proliferation, migration, and differentiation of endogenous stem and progenitor cells. Here, we compared gene expression changes following the selective loss of two retinal neuronal subtypes, nyctalopin-expressing bipolar cell interneurons (35) and rod photoreceptors (36). Whole eye samples were collected over twelve time points encompassing the loss and replacement of the targeted cell types. Differential gene expression analysis across a total of 138 microarrays revealed both shared and cell-specific profiles. Cell-specific expression changes and signaling pathways predominated at early and late time points with shared gene profiles peaking at 32 hours after induction of cell loss (21\% of differentially expressed genes). While cell-specific mechanisms were expected to be prevalent during late phases, corresponding to fate-biased progenitor cell differentiation, dominance at early time points suggests that MG stem cell activation can occur via pathways unique to the cell type lost. In turn, this suggests the nature of degenerative events informs the regenerative process, consistent with lineage tracing experiments demonstrating fate-biased regeneration following selective cell loss. Given the relevance to numerous degenerative diseases, greater understanding of the mechanisms regulating fate-biased regeneration, i.e., stem cell responses to selective cell loss, will aid the development of targeted regenerative therapeutics. 


\section{Results}

\section{Specificity of NTR/MTZ-induced cell death and regeneration assessments}

To investigate mechanisms underlying the death and regeneration of retinal cell types, two transgenic zebrafish lines expressing cell ablation-reporter moieties in bipolar or rod photoreceptor cells were used: 'NTR-bipolar' (roy ${ }^{a 9 /+} ; q 16 a^{+/-} ; q 16 b^{+/} ; c 264^{+/-} ;(39)$ ) and 'NTR-rod' (roy ${ }^{a 9 /+}$; gmc500 $\left.{ }^{+-} ;(36)\right)$. We first confirmed that NTR transgene expression was specific to the targeted cell types within the retina. Prior studies have shown that NTR-bipolar transgene expression is largely restricted to bipolar cells with labeling of the pineal gland (epiphysis) as well $(35,40)$. For the NTR-rod line, immunohistochemistry confirmed that YFP-NTR expression was restricted to rod cells (Supp. Fig 1). Immunostaining showed that YFP-NTR expressing cells co-labeled with a rod-specific outer segment marking antibody, zpr-3 (41, 42); Supp Fig. 1a-a"), and interdigitated with a cone-specific antibody, zpr-1 $(41,43)$; Supp Fig. 1b-b"). These results confirm previous reports for this promoter element (44).

We next verified that $24 \mathrm{hr} 10 \mathrm{mM}$ Mtz prodrug treatments induce selective loss of NTRexpressing cells and that regeneration occurs upon MTZ washout. Confocal imaging was used to follow this process in individual NTR-bipolar and NTR-rod larvae (Fig. 1a-a' and b-b', respectively). After Mtz treatment (6-7 dpf), NTR+ cells appeared to fragment and degenerate in comparison to pre-treatment images. Four days after Mtz was removed (11 dpf), both NTR+ bipolar and rod cells were observed to reappear (Fig. 1a', b' '). Retinas were then TUNEL stained (terminal deoxynucleotidyl transferase (TdT)-mediated dUTP nick end labeling, cell death marker (45)) to confirm that 1) ablated cell types underwent cell death, and 2) ensure Mtz-induced losses were limited to the targeted cell types. Both bipolar (Fig. 1c, d) and rod (Fig. 1e, f) cells were appropriately labeled following TUNEL staining as expected. Quantification showed a significant increase in cell death following Mtz exposures (Fig. 1g).

\section{Characterization of retinal stem cell niche responses to bipolar and rod cell death}

As our NTR-rod line consistently labels the majority of rod photoreceptor cells (Supp. Fig. 1, Fig. 1), we anticipated that rod cell loss would trigger proliferation in both the INL and ONL. The NTR-bipolar line uses the Gal4/UAS bipartite system to express two different transgenes, a membrane-tagged YFP and an NTR-mCherry fusion protein. In this background NTR-mCherry expression in the NTR-bipolar larvae is sporadic, with the number of NTR+ cells varying from low ( $\sim 25$ cells/retina) to high (hundreds of cells/retina). To account for this variability, low-mid level expressing NTR-bipolar larvae ( $\sim 100$ targeted cells) were visually selected for this and subsequent microarray studies.

To determine which retinal stem cell niche(s) responded in the two cell loss paradigms, Mtz-treated retinas were immunolabeled with proliferative cell nuclear antigen (PCNA). The data showed cellspecific trends, with a bias toward INL stem/progenitor proliferation following bipolar cell loss (Fig. 1h, i) and relatively more ONL progenitors responding to rod loss (Fig. 1j, k). More proliferation was also seen in ablated NTR-rod lines ( $\sim 14$ PCNA+ cells/retinal slice), as opposed to ablated NTR-bipolar fish ( $\sim 5$ PCNA+ cells/retinal slice). This difference was due to elevated levels of ONL progenitor proliferation following rod ablation; the amount of INL proliferation was similar in both paradigms (Fig. 11). Proliferation within the INL in Mtz-treated NTR-rod lines was typically clustered near the periphery where the density of cell loss was highest (Fig. 1k), 
consistent with increased rod cell death activating MG stem cells (46). ONL localized division was also observed following bipolar cell ablation (Fig. 1i).

Time series imaging was used to compare pre- and post-MTZ treated individual larvae (Supp Fig. 2). When NTR-rod lines were treated with DMSO (control), no substantial loss of photoreceptors was observed (Supp Fig. 2a, a'). Conversely, upon treatment with 10mM Mtz for $24 \mathrm{~h}$ a rapid loss of photoreceptors occurred (Supp Fig. 2b, b'). Subsequent wash out and recovery over the next 5 days revealed a robust regenerative response to replenish lost photoreceptors at $12 \mathrm{dpf}$ (Supp Fig. $3 \mathrm{~b}$ '). Similar treatment of NTR-bipolar lines produced equivalent results; DMSO (control) resulted in no loss of bipolar interneurons (Supp Fig. 2c-c' ') while $24 \mathrm{hr}$ Mtz treatment (6 to $7 \mathrm{dpf}$ ) and washout led to rapid bipolar cell loss and subsequent regeneration, respectively (Supp Fig. 2dd').

\section{Microarray analysis of two cell-specific regeneration paradigms}

For time-resolved differential gene expression analysis, eyes were collected from NTR-bipolar and NTR-rod larvae treated \pm MTZ across a total of 12 time points spanning cell loss and replacement

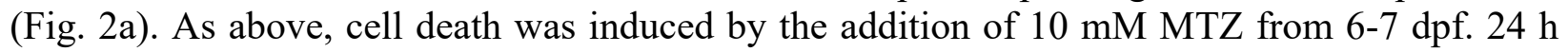
MTZ treatments were followed by wash out and recovery from days 7-16 dpf (Fig. 2a). To assess retinal stem cell responses to cell loss, early time points were emphasized for RNA analysis. Bulk RNA samples were collected from larval retinas isolated every $8 \mathrm{hr}$ after the onset of MTZ exposures from $0-48 \mathrm{~h}$ (i.e., $24 \mathrm{~h}$ into recovery), then at 60, 72, 96, 144, and $240 \mathrm{~h}$. Eight-hour intervals were used from $0-48 \mathrm{hrs}$ to minimize detection of gene expression changes due solely to circadian cycles. This process was repeated a total three times for each paradigm (Supp. Table 1). Samples passing RNA quality checks were then used for microarray expression analysis. All microarrays were normalized to each other using Robust multi-array average (RMA), a normalization method designed to decrease noise when comparing multiple arrays (47).

\section{Genetic differences following regeneration of uniquely targeted neurons}

Using a 1.5-fold difference cutoff and a p-value $<=0.05$, a total of 2,463 genes were observed to change expression following retinal cell ablation. 696 and 1085 were unique to the rod and bipolar cell ablation paradigms, respectively, and 682 being shared between paradigms (Fig. 2b). The number of highly differentially expressed genes (2-fold cutoff) per time point was calculated for each paradigm and plotted relative to the total number of genes meeting that criteria (Fig. 2c). The NTR-rod regeneration paradigm had a peak in the number of highly differentially expressed genes at $60 \mathrm{hrs}$ post-MTZ (Fig. 2c). Corresponding changes in gene expression associated with the NTRbipolar paradigm peaked at $24 \mathrm{hrs}$ post-MTZ (Fig. 2c).

Several analyses were performed to identify signaling pathways associated with specific time points (Fig. 2d, Supplementary Figs. 4-14) or time-resolved expression patterns (Fig. 3). First, to assess signaling pathways associated with retinal stem cell activation, Ingenuity Pathway Analysis (IPA) was used to interrogate gene changes at $8 \mathrm{hrs}$ after MTZ exposure (Fig. 2d). This analysis revealed differential regulation of pathways associated with immune system reactivity (Interferon, Granzyme A and IL-6 signaling), MG activation (JAK/STAT signaling), phototransduction and other related pathways (Fig. 2d). Most pathways showed simultaneous upregulation and downregulation, however, some were solely upregulated (e.g., IL-6 and hormone-like cytokine 
signaling). In addition, some pathways were differentially regulated between the two paradigms (e.g., interferon and granzyme A signaling).

Quantitative PCR was used to validate the microarrays, as microarray data can be noisy (64) and the use of certain analytical methods, i.e. selecting $>1.5$-fold change or $p$-value cutoffs, can significantly alter data interpretation (65). The JAK/STAT and Wnt pathways were used for qRTPCR validation as both were identified as major networks involved in regeneration of these cell types previously $(6,66)$. Temporal expression trends were utilized for validation when comparing microarray data to qRT-PCR results as differences in normalization methods have the potential to impact overall expression levels (51). Only minor differences were observed between the microarray and qRT-PCR data suggesting the microarray data accurately recapitulates gene expression changes (Supp Fig. 3).

\section{Genetic differences following regeneration of uniquely targeted neurons}

We performed KEGG and GO analyses on microarray data at each time point from 8-240 h postMTZ in order to identify signaling pathways that were responsible for activating MG and Müller glia Progenitor Cells (MGPC). For both rod and bipolar paradigms, volcano plots were produced to show log-fold changes in gene expression (Supp. Fig. 4-14a). Then, the total number of differentially and commonly regulated genes were plotted (Supp. Fig. 4-14b). Lastly, dot plots were created to show significant KEGG functional regulation events (Supp. Fig. 4-14 c). Then, to gain organized perspective on dynamically expressed genes at crucial time points, we combined GO and KEGG pathway analysis with a focus on expression changes (up and down) early and late in the regeneration process. Early regeneration was defined as 24 and $32 \mathrm{~h}$ postMTZ, and late as 60 and $72 \mathrm{~h}$. This yielded four patterns of gene expression (e.g. early + up, early + down, late + up, and late + down) and further layered into genes/pathways dynamically regulated specifically in NTR-rod or NTR-bipolar ablation or shared by both paradigms (Fig. 3a, $\mathrm{b}, \mathrm{c})$.

Pattern 1, genes upregulated early in regeneration yielded similar peaks at 24 and $32 \mathrm{~h}$ for NTRrod and NTR-bipolar paradigms (Fig. 4a). GO analysis showed common negative regulation of insulin signaling, JAK/STAT pathway, and protein kinase activity, while paradigm specific terms included cell differentiation, transcription and regulation of cell proliferation in rods and iron transport in bipolar neurons (Fig. 4b, c). Specific genes identified by GO included socs1a, socs3a, and cish (Fig. 4d). KEGG pathway analysis isolated JAK/STAT signaling and glutathione metabolism in rods and Cell Adhesion Molecules and Fructose/Mannose metabolism in bipolar cells (Fig. 4e). Pattern 2, Genes downregulated early in regeneration yielded "valleys" at $24+32 \mathrm{~h}$ in expression (Fig. 5a). Shared GO terms included only visual perception while paradigm specific GO terms included axon extension in rods, and in bipolar cells transcription, fin regeneration, and nervous system development (Fig. 5b, c). Irbp was a commonly downregulated gene at this time (Fig. 5d). KEGG analysis highlighted metabolism pathways in rods, circadian rhythm, splicing, and MAPK pathway in bipolar cells and shared differential regulation of glycolysis and gluconeogenesis (Fig. 5e).

Later in regeneration, more commonly differentially regulated genes were measured (235 compared to 152) (Fig. 3C). Pattern 3, shared GO terms upregulated at these later timepoints included apoptosis and MOMP (mitochondrial outer membrane permeabilization) while paradigm 
specific terms included transcription for rods, and autophagy, mitophagy, and MAPKKK activation in bipolar cells (Fig. 6a-c). Shared specific genes included bnip3 and bnip3b (Fig. 6d). KEGG analysis additionally isolated shared lysosomal pathway regulation, rod-specific pathways included tight junctions and insulin signaling while bipolar cells included p53, toll-like receptor, cell cycle, MAPK signaling and more (Fig. 6d). Lastly, Pattern 4, genes downregulated late in regeneration, showed the most commonalities between the two cell type paradigms and exhibited similar 60+72 h valleys (Fig. 7a). Shared GO terms included DNA replication and protein folding while the rod specific changes included tRNA aminoacylation, response to light, biosynthesis, translation and more. Bipolar-specific GO terms included cell cycle, retinal development, and DNA damage response. Numerous specific shared genes were identified including mcm4, rbb4, and more (Fig. 7b-d). KEGG analysis identified 11 shared pathways including biosynthesis, replication, cell cycle, mismatch repair, and more. Rod-specific KEGG terms included RNA degradation and various metabolic pathways while bipolar-specific terms included p53 signaling, different metabolic pathways, the TCA cycle, and more (Fig. 7e).

\section{Discussion}

Promotion of retinal regeneration would provide a transformative therapeutic approach for blinding diseases/conditions. Recent studies in zebrafish have identified several genes involved in retinal regeneration: asclla (48), lin28a, sox2 (49), stat3 (50) and more (51-53). However, the methods used for these studies often incurred the loss of multiple retinal cell types. As retinal diseases are typically linked to the loss of specific cell types (54) - and "fate biased" regenerative processes are associated with selective cell ablation in zebrafish (8-11) - additional insights may arise from assessing transcriptomic responses to targeted retinal cell loss. Cell-specific zebrafish retinal disease models are available $(55,56)$, however these involve spontaneous/constitutive cell death, thus compromising the ability to study the regenerative process in isolation from cell loss. To overcome this, we utilized the NTR-based system of inducible cell ablation (33) to investigate potential cell-specific regenerative mechanisms. Specifically, we compared transcriptomic responses between the targeted loss of rod photoreceptors (NTR-rod) versus bipolar interneurons (NTR-bipolar; Fig. 1a-a', b-b'). Proliferative responses were assessed as prior NTR-based studies showed that the extent of rod cell loss can trigger responses in two distinct stem/progenitor cell responses; i.e., low rod loss incurring only rod-committed progenitor cell proliferation in the ONL while extensive rod loss was associated with proliferation in the ONL and INL-localized MG cells as well (46). The data confirmed that our rod cell ablation paradigm initiated a proliferative response in both the INL and ONL (Fig. 1k), akin to the bipolar cell ablation paradigm (Fig. 1i), demonstrating that both paradigms trigger MG proliferation. Any differences in gene expression observed between these paradigms cannot therefore be explained solely by differential stem cell responses; i.e., activation of only rod-committed progenitors in the ONL for the NTR-rod paradigm.

To comprehensively characterize rod and bipolar cell regeneration a total of 138 microarrays were used, spanning 12 time points encompassing cell loss and replacement for both paradigms (Fig. 2a). Prior microarray studies had identified genes associated with MG activation (53) and networks activated following various retinal lesions $(38,57)$. By expanding the number of time points analyzed, we sought to identify genes/pathways associated with: 1) stem cell activation, 2) stem cell dedifferentiation, 3) stem/progenitor cell proliferation, and 4) cell-specific differentiation. We 
emphasized early timepoints (every $8 \mathrm{~h}$ from $0-48 \mathrm{~h}$ ) in an effort to isolate genes linked to the initiation of fate biased regenerative processes. A total of 682 genes showed shared differential expression patterns. Rod cell loss and regeneration was associated with 696 paradigm-specific gene changes, while the bipolar paradigm was linked to 1085 unique genes (Fig. 2b). Ingenuity Pathway Analysis (IPA, QIAGEN), Gene Ontology (GO) and Kyoto Encyclopedia of Genes and Genomes (KEGG) pathway analysis (58) were used to assess functionally correlated gene expression changes. Data for each timepoint from 8-240 h post-ablation was first assembled (Supp Fig. 4-14) before moving to analysis of four coordinated expression patterns (Fig. 3).

IPA was used to compare data from the earliest time point analyzed once cell death had been induced, $8 \mathrm{~h}$ after administration with MTZ, in keeping with our emphasis on early events involved in activating stem cell responses to retinal cell loss. This analysis revealed differential regulation of several pathways involved in immune system regulation, e.g., interferon, prolactin, and granzyme A signaling, as well as shared upregulation of IL-6 and hormone-like cytokine signaling. Many of these pathways converge on JAK/STAT signaling, a shared pathway also revealed by IPA that appeared to be similarly regulated across cell loss paradigms. This data underscores the emerging importance of the immune system in neural regenerative processes, consistent with recent findings implicating microglia/macrophages in regulating retinal regeneration $(32,52,59-$ $61)$.

Among GO terms, JAK/STAT arose again. JAK/STAT is a major signaling pathway involved in cell migration (62), proliferation (50), apoptosis (63) and immune system responses (64). Activation of the pathway is initiated by extracellular cytokines, growth factors, or interferon (IFN) binding to their respective receptors $(65,66)$, in response to oxidative stress (67), and also in response to retinal damage $(50,68)$. While factors involved in the JAK/STAT pathway appear to be tightly regulated, deregulation has been linked to a variety of melanomas making it a prime target for tumor suppression studies and cancer therapies $(69,70)$. As previously observed (51), upregulation of genes in the JAK/STAT pathway, particularly stat 3 activation, occurred at $8 \mathrm{~h}$ post ablation of both rod or bipolar cells. At $24 \mathrm{~h}$ in our time series, upregulation of socs $1 a$, socs $3 a$, and cish occurred in both paradigms (Fig. 2d, 4b, d). These genes belong to the Suppression of Cytokine Signaling (SOCS) family and are regulators of immune system responses and JAK/STAT signaling specifically (71). Intriguingly, socs $3 a$ activation has been associated with stat 3 activation and noted as a mediator of hair and liver cell regeneration in zebrafish through activation of resident stem cells and progenitor differentiation $(72,73)$. Interesting, the JAK/STAT pathway came up twice more for early upregulation in the rod paradigm specifically (Fig. 4c, e), suggesting additional important relative to bipolar cell processes. Genes downregulated at this early timepoint, Pattern 2, include those associated with visual perception, confirming loss of the targeted retinal cell types (Fig. 5).

Later in our time series, at 60 and $72 \mathrm{~h}$ post-MTZ treatment, GO and KEGG pathway analysis primarily identified changes in genes involved in the cell cycle, cell death, and proliferation. Specifically, genes upregulated in both rods and bipolar cells at these times (e.g. bnip3, zgc:73226) are implicated in apoptosis and mitochondrial outer membrane permeabilization (MOMP; Fig. 6b, d). Bipolar cell loss was additionally associated with mitophagy, autophagy, and autophagosome assembly (Fig. 6C). The bnip3 gene is in the Bcl-2 family and thought to increase mitochondrial turnover via autophagy (74), bnip3 and autophagy have been well-studied in the context of muscle- 
repair, where inhibition of autophagy leads to defective regeneration in zebrafish (75). Our final pattern, genes downregulated later in regeneration, showed the most similarities between the two paradigms. Implicated pathways DNA replication and initiation, and protein folding (Fig. 7b, d). Shared KEGG pathways included DNA replication, cell cycle, mismatch repair, numerous metabolic pathways and more (Fig. 7e). Bipolar cells had additional implicated pathways of nucleotide and base excision repair as well as p53 signaling (Fig. 7e).

Our study revealed frequent dynamic regulation of glutathione, insulin and glucose metabolism during the regeneration of both cell types. Early on, shared upregulation of glutathione metabolism occurred (Fig. 4e); this can be used as a measure of increased oxidative stress which has been observed to increase greatly following retinal damage in both zebrafish and mice $(53,76,77)$. Additionally, high glutathione levels are thought to be critical for maintaining stem cell functions $(78,79)$. Glutathione metabolism was subsequently downregulated in rods specifically (Fig. 7e). Shared early negative regulation of glycolysis/gluconeogenesis genes was observed (Fig. 5d); consistent with findings that a shift in energy production occurs in stem cell differentiation in the retina $(80,81)$. In the bipolar paradigm specifically, we identified early downregulation of both the MAPK pathway (16 genes) and insulin resistance ( 8 genes, Fig. 5E), while later MAPK genes were then upregulated in the bipolar cell paradigm (Fig. 6c), suggesting MAPK expression is tightly regulated in these cells during regeneration. Downregulation of insulin resistance is in line with findings that insulin and igf- 1 expression is key for forming and proliferating MG progenitor cells $(82,83)$. The specificity of this phenomena to bipolar regeneration demonstrates utility of cell-type specific regeneration studies.

In conclusion, transcriptomic comparison of two selective retinal cell ablated paradigms led to the identification of shared and paradigm-specific gene expression changes. Temporally coordinated patterns emerged that revealed coordinated gene changes among major signaling pathways. Shared implication of JAK/STAT signaling is in agreement with prior studies using widespread retinal damage paradigms, including early activation of stat 3 . Early stem cell activation phases were associated with multiple genes from the SOCS family, known regulators of stat 3 activation linked to regeneration of hair and liver cells previously $(72,73)$ and now in retinal regeneration. Shifts in glutathione metabolism also appear to be critical for MG based regeneration, similar to what was observed in stem cells previously as a result of oxidative stress $(78,79)$; in our analysis this was shared between both paradigms. Later in regeneration, activation of DNA replication, cell cycle and protein synthesis genes were observed. In addition, we noted upregulation of apoptotic and autophagic processes, previously linked to zebrafish regeneration studies (75). Uniquely, side-byside comparison of two cell-specific ablation models allowed us to reveal paradigm-specific factors/pathways, akin to recent cross-species comparisons to photoreceptor versus inner retinal neuron ablation (84). Paradigm-specific factors/pathways included: enhanced JAK/STAT regulation for rod cell regeneration and insulin resistance, MAPK and p53 signaling for bipolar cell regeneration. It is likely that novel pathways were easier to associate with bipolar cell regeneration due to the fact that this paradigm has been comparatively understudied compared to rod photoreceptor regeneration. These findings expand upon previous studies demonstrating that selective retinal cell loss can trigger a fate biased regenerative responses (8-11), and provide potential insights useful for developing selective cell regeneration therapies. Collectively, these findings emphasize that regeneration need not always "recapitulate development", particularly for paradigms that mimic the selective cell loss attending most neurodegenerative diseases. 


\section{Materials and Methods}

\section{Aquaculture}

Zebrafish were maintained under standard environmental conditions $\left(\sim 28^{\circ} \mathrm{C}, 14 \mathrm{hr}: 10 \mathrm{hr}\right.$ light:dark cycle) under an approved ACUC animal use protocol. Larvae were fed paramecia starting at 5 days post-fertilization (dpf), then paramecia and Artemia salina from 9-16 dpf. Late stage larvae (e.g., $8 \mathrm{dpf}$ ) slated for imaging were kept in 12 well plates in $5 \mathrm{ml}$ of system water until $12 \mathrm{dpf}$ after which they were sacrificed.

\section{Transgenic lines}

A bipartite Gal4/UAS (85) transgenic zebrafish line derived with a 1,482 bp promoter element from the nyctalopin (nyx) gene (86, 87), $\operatorname{Tg}($ nyx:Gal4-VP16)q16a, and a Gal4-driven reporter, $(U A S: G A P-Y F P) q 16 b$, expresses membrane-tagged yellow fluorescent protein (YFP) in a subpopulation of bipolar cells (40). Crosses to a UAS-reporter/effector line facilitating prodruginduced cell ablation, $\operatorname{Tg}\left(U A S-E 1 b: N f_{S B}-m\right.$ Cherry)c264 (88), resulted in triple transgenic fish expressing an NTR-mCherry fusion protein in subsets of nyx-promoter targeted bipolar cells (q16a;q16b;c264; hereafter, NTR-bipolar lines). A transgenic line derived with a $3.7 \mathrm{~kb}$ promoter element from the rhodopsin (rho) gene (kind gift of Dr. Shoji Kawamura) (44) expresses a YFPNTR fusion protein in rod photoreceptor cells, $T g($ rho: YFP-Eco.NfsB)gmc500 (36) (hereafter, NTR-rod lines). Retinal morphology was monitored during imaging studies by crossing both NTR lines with $T g$ (pax6-DF4:gap43-CFP)q01 which expresses membrane tagged CFP throughout the retina (see Fig. 1A-B) (89). Transgenic fish were propagated in the roy orbison (aka, mpv17 ${ }^{a 9 / a 9}$, hereafter $\left.\operatorname{roy}^{a / a 9}\right)(90,91)$ pigmentation mutant background to facilitate intravital imaging (92). However, for differential gene expression studies, to minimize expression variance due to differences in transgene copy number, outcrosses to an AB wildtype strain (WT) were performed to generate heterozygotes.

\section{MTZ treatment}

Transgenic NTR-bipolar and NTR-rod larvae were separated into two equal sized groups at 6 dpf: 1) non-treated controls (Cntl); and 2) those treated with $10 \mathrm{mM} \mathrm{MTZ}$ for $24 \mathrm{hr}$ to induce cell death, from 6-7 dpf. After MTZ treatment, fish were rinsed into system water and fed paramecia until sacrificed.

\section{mRNA isolation and preparation}

Whole eye mRNA was prepared across twelve time points: t0 (just prior to MTZ exposure); $t 8$, $\mathrm{t} 16$, and $\mathrm{t} 24 \mathrm{hr}$ (during MTZ treatment); and t32, t40, t48, t60, t72, t96, t144, and $\mathrm{t} 240 \mathrm{hr}$ (after MTZ removal during recovery (Fig. 2A). Sample collection started at 8:00 a.m. on the day larvae reached 6 dpf. At each time point, 16 eyes from 8 larvae were isolated per condition. To isolate eyes, larvae were euthanized in $0.4 \%$ tricaine for 5 mins followed by cervical transection. Whole eye pairs were manually isolated under an Olympus SZX16 epifluorescence stereoscope and immediately placed into a chilled/sterile $1.5 \mathrm{ml}$ tube with $50 \mu 1$ of TriZOL (Life Technologies); samples were then stored at $-80^{\circ} \mathrm{C}$ until extraction. This protocol was followed for three biological repeats per condition/time point for NTR-rod and NTR-bipolar lines. Samples were batch extracted and processed following the final time. All samples were processed in the Medical College of 
Georgia (MCG, former Mumm lab location) Integrated Genomics Core for mRNA isolation, cDNA library construction, and microarray hybridization.

\section{Microarrays}

GeneChip Zebrafish Genome Microarrays (Affymetrix) were processed by the MCG Genomic Core. Each run involved sibling zebrafish and consisted of 23 RNA samples across each regenerative paradigm - 1 pretreatment, and 11 time points from the control and MTZ treatment groups. RNA sample quality was assessed by concentration and the RNA Integrity Number (RIN), an algorithm that assigns integrity values to RNA measurements (93). Only samples attaining a minimal concentration of $\geq 100 \mathrm{ng} / \mu \mathrm{L}$ ( $5 \mu \mathrm{L}$ total) and a RNA integrity number (RIN) value of $\geq 8$ were used for microarray hybridizations (93). Runs where a minimum of 21 of 23 samples were deemed of good quality were utilized in the microarray studies. Three runs were processed per regenerative paradigm resulting in six microarray sets (Supp. Table 1) and a total 138 microarrays overall.

\section{Tissue preparation and immunohistochemistry}

For immunohistochemistry, larval zebrafish were euthanized, fixed in 4\% paraformaldehyde (PFA) for $8 \mathrm{hr}$, washed three times in 1x PBS (phosphate buffered saline; EMD Millipore) for $30 \mathrm{~min}$ and stored at $4^{\circ} \mathrm{C}$. Samples were mounted in cryogel embedding medium within the next 2-4 days, frozen in liquid nitrogen then stored at $-80^{\circ} \mathrm{C}$ until sectioned in the lateral plane at $25 \mu \mathrm{m}$ thickness with a cryostat. Sliced sections were collected on standard microscope slides and stored at $4{ }^{\circ} \mathrm{C}$.

For immunolabeling, slides were air dried at room temperature for $\sim 1 \mathrm{hr}$, rinsed in $1 \mathrm{xPBS}$ and then re-fixed with 4\% PFA for 15 min. PBST rinses (1xPBS $+0.1 \%$ Tween20, Fisher Scientific) were conducted to remove trace PFA followed by $5 \mathrm{~min}$ antigen retrieval with SDS (1\% Sodium Dodecyl Sulfate; Fisher Scientific) in PBS. The blocking phase was performed with 3\% goat serum in PBDT (1xPBS, 1\% BSA, 1\% DMSO, 0.1\% TritonX-100) for 30 min and incubated with primary antibody $/ 1 \%$ goat serum/PBDT overnight at $4^{\circ} \mathrm{C}$. The next morning, slides were rinsed in PBST, stained with secondary antibody/PBDT $\sim 1 \mathrm{hr}$ in a light protected humidity chamber and coverslipped $(22 \times 50 \mathrm{~mm}$, Fisher Scientific). PBST rinses removed unbound secondary antibody. Samples were protected with Vectashield + DAPI (Vector Laboratories) and cover-slipped (24x50 $\mathrm{mm}$, Fisher Scientific).

Primary antibodies included: mouse anti-PCNA monoclonal antibody (1:1000, clone PC10; Sigma Aldrich), rabbit anti-Caspase-3 monoclonal antibody (1:500, clone C92-605; BD Biosciences), Click-iT Tunel Alexa Fluor 647 (1:500; Life Technologies), mouse anti-zpr-1 monoclonal antibody (1:750; ZIRC), mouse anti-zpr-3 monoclonal antibody (1:750; ZIRC), mouse anti-ZS-4 monoclonal antibody (1:750; ZIRC). Secondary antibodies included: anti-mouse Alexa Fluor 430 (1:500; Life Technologies), anti-mouse Alexa Fluor 635 (1:500; Life Technologies), anti-rabbit Alexa Fluor 430 (1:500; Life Technologies).

\section{Cell counting and analysis}

Images were collected with an Olympus FV1000 Confocal Microscope (405, 440, 488, 515, 559, and $635 \mathrm{~nm}$ laser lines). Stacked confocal images were obtained using a 40x oil immersion objective with a $2.5 \mu \mathrm{m}$ step size, $130 \mu \mathrm{m}$ aperture, and $10 \mu \mathrm{m}$ total depth. Five sections were collected per retina centered around the optic nerve. Images (Olympus .OIB format) were analyzed using ImageJ. Manual cell counts were averaged across sections per retina and averaged within 
each group. Student's t-test and One-way ANOVA were used for statistical comparisons between groups.

\section{Sample quality and differential expression analysis}

Data were collected from 138 Genechip Zebrafish Genome arrays (Affymetrix) across four conditions: NTR-rod cntrl, NTR-rod MTZ, NTR-bipolar cntrl, and NTR-bipolar MTZ. The Bioconductor suite in the R statistical software (94), LIMMA (95), Carmaweb (96), and Microsoft Access were used for data processing, differential expression testing and storage. Prior to statistical analysis, Robust Multi-array Average (RMA) pre-processing routine (47) and Quality control assessments (97) were used for background correction, normalization and quality control. A set of differentially expressed genes was derived using LIMMA with a false discovery rate adjusted pvalue of 0.05 . Differentially expressed genes were defined as those expressing a 1.5-fold up or downregulation at any timepoint in regeneration. Pathway analysis was performed using Ingenuity Pathway Analysis (IPA), Gene Ontology (GO) and KEGG pathway analysis. A p value below 0.05 was used throughout as a cutoff for significance.

\section{qPCR processing}

Extracted mRNA samples were reverse transcribed (Qiagen Omniscript RT kit, Qiagen) and stored at $-20^{\circ} \mathrm{C}$. Samples were run in triplicate using the BioRad iQ SYBR Green Supermix (BioRad) in iCycler IQ 96 well PCR plates (Bio-Rad) on a BioRad iCycler equipped with an iCycler iQ Detection System. The protocol consisted of three phases: 1) $92^{\circ} \mathrm{C}$ for $\left.10: 00 \mathrm{~min}, 2\right) 50 \mathrm{x} 92^{\circ} \mathrm{C}$ for $00: 15 \mathrm{~min}, 60^{\circ} \mathrm{C}$ for $\left.01: 00 \mathrm{~min}, 3\right) 81 \times 55^{\circ} \mathrm{c} \rightarrow 95^{\circ} \mathrm{c}$ for $00: 10 \mathrm{~min}$. $\beta$-actin served as the house keeping gene and $2^{-\Delta \Delta C T}$ method was used for normalization to ensure equal amounts of cDNA for comparisons. qPCR primers were designed using the online tool QuantPrime.

\section{Acknowledgements}

We thank the MCG Vision Discovery Institute of Augusta University for funding this work. Additional funding supporting this work included: R01EY022810 (JSM). The authors also wish to thank Drs. Grzegorz Rempala and Daniel Linder for initial assistance in analyzing this data using Bayesian statistics, as well as members of the Mumm and Qian labs for helpful discussions.

\section{References}

1. Tsonis P a (2002) Regenerative biology: the emerging field of tissue repair and restoration. Differentiation 70(8):397-409.

2. Simon A, Tanaka EM (2012) Limb regeneration. Wiley Interdiscip Rev Dev Biol 2(2):291300.

3. Nowotarski SH, Sánchez Alvarado A (2016) Widening perspectives on regenerative processes through growth. npj Regen Med 1(1):16015.

4. Lenkowski JR, Raymond PA (2014) Müller glia: Stem cells for generation and regeneration of retinal neurons in teleost fish. Prog Retin Eye Res 40:94-123.

5. Goldman D (2014) Müller glial cell reprogramming and retina regeneration. Nat Rev Neurosci 15(7):431-442. 
6. Gorsuch R a, Hyde DR (2014) Regulation of Müller glial dependent neuronal regeneration in the damaged adult zebrafish retina. Exp Eye Res 123:131-40.

7. Elsaeidi F, et al. (2018) Notch suppression collaborates with Ascl1 and Lin28 to unleash a regenerative response in fish retina, but not in mice. $J$ Neurosci. doi:10.1523/JNEUROSCI.2126-17.2018.

8. Fraser B, DuVal MG, Wang H, Allison WT (2013) Regeneration of Cone Photoreceptors when Cell Ablation Is Primarily Restricted to a Particular Cone Subtype. PLoS One. doi:10.1371/journal.pone.0055410.

9. D'Orazi FD, Suzuki SC, Darling N, Wong RO, Yoshimatsu T (2020) Conditional and biased regeneration of cone photoreceptor types in the zebrafish retina. J Comp Neurol. doi:10.1002/cne.24933.

10. Ng Chi Kei J, Currie PD, Jusuf PR (2017) Fate bias during neural regeneration adjusts dynamically without recapitulating developmental fate progression. Neural Dev. doi:10.1186/s13064-017-0089-y.

11. DuVal MG, Allison WT (2017) Impacts of the retinal environment and photoreceptor type on functional regeneration. Neural Regen Res. doi:10.4103/1673-5374.202930.

12. Braisted JE, Essman TF, Raymond P a (1994) Selective regeneration of photoreceptors in goldfish retina. Development 120(9):2409-19.

13. Vihtelic TS, Hyde DR (2000) Light-Induced Rod and Cone Cell Death and Regeneration in the Adult albino Zebrafish ( Danio rerio ) Retina ABSTRACT :

14. Thomas JL, Nelson CM, Luo X, Hyde DR, Thummel R (2012) Characterization of multiple light damage paradigms reveals regional differences in photoreceptor loss. Exp Eye Res. doi:10.1016/j.exer.2012.02.004.

15. Thomas JL, Thummel R (2013) A novel light damage paradigm for use in retinal regeneration studies in adult zebrafish. $J$ Vis Exp.

16. Maier W, Wolburg H (1979) Regeneration of the Goldfish Retina After Exposure to Different Doses of Ouabain. Cell Tissue Res 118:99-118.

17. Fimbel SM, Montgomery JE, Burket CT, Hyde DR (2007) Regeneration of inner retinal neurons after intravitreal injection of ouabain in zebrafish. $J$ Neurosci 27(7):1712-24.

18. Hitchcock PF, Lindsey Myhr KJ, Easter SS, Mangione-Smith R, Jones DD (1992) Local regeneration in the retina of the goldfish. $J$ Neurobiol 23(2):187-203.

19. Cameron D a, Easter SS (1995) Cone photoreceptor regeneration in adult fish retina: phenotypic determination and mosaic pattern formation. J Neurosci 15(3 Pt 2):2255-71.

20. Johns PR, Fernald RD (1981) Genesis of rods in teleost fish retina. Nature. doi:10.1038/293141a0.

21. Raymond PA, Rivlin PK (1987) Germinal cells in the goldfish retina that produce rod photoreceptors. Dev Biol. doi:10.1016/0012-1606(87)90338-1.

22. Wu DM, et al. (2001) Cones regenerate from retinal stem cells sequestered in the inner 
nuclear layer of adult goldfish retina. Invest Ophthalmol Vis Sci 42(9):2115-24.

23. Hitchcock PF, Raymond PA (2004) The Teleost Retina as a Model for Developmental and Regeneration Biology. Zebrafish. doi:10.1089/zeb.2004.1.257.

24. Jorstad NL, et al. (2017) Stimulation of functional neuronal regeneration from Müller glia in adult mice. Nature. doi:10.1038/nature23283.

25. Anlezark GM, et al. (1992) The bioactivation of 5-(aziridin-1-yl)-2,4-dinitrobenzamide (CB1954)--I. Purification and properties of a nitroreductase enzyme from Escherichia coli-a potential enzyme for antibody-directed enzyme prodrug therapy (ADEPT). Biochem Pharmacol 44(12):2289-95.

26. Knox RJ, Friedlos F, Sherwood RF, Melton RG, Anlezark GM (1992) The bioactivation of 5-(aziridin-1-yl)-2,4-dinitrobenzamide (CB1954)--II. A comparison of an Escherichia coli nitroreductase and Walker DT diaphorase. Biochem Pharmacol 44(12):2297-301.

27. Sunters A, Baer J, Bagshawe KD (1991) Cytotoxicity and activation of CB1954 in a human tumour cell line. Biochem Pharmacol 41(9):1293-8.

28. Williams EM, et al. (2015) Nitroreductase gene-directed enzyme prodrug therapy: insights and advances toward clinical utility. Biochem J 471(2):131-153.

29. Bridgewater JA, et al. (1995) Expression of the bacterial nitroreductase enzyme in mammalian cells renders them selectively sensitive to killing by the prodrug CB1954. Eur $J$ Cancer 31A(13-14):2362-2370.

30. White DT, Mumm JS (2013) The nitroreductase system of inducible targeted ablation facilitates cell-specific regenerative studies in zebrafish. Methods 62(3):232-240.

31. Mathias JR, Zhang Z, Saxena MT, Mumm JS (2014) Enhanced cell-specific ablation in zebrafish using a triple mutant of escherichia coli nitroreductase. Zebrafish. doi:10.1089/zeb.2013.0937.

32. White DT, et al. (2017) Immunomodulation-accelerated neuronal regeneration following selective rod photoreceptor cell ablation in the zebrafish retina. Proc Natl Acad Sci U S A. doi:10.1073/pnas.1617721114.

33. Curado S, et al. (2007) Conditional targeted cell ablation in zebrafish: A new tool for regeneration studies. Dev Dyn. doi:10.1002/dvdy.21100.

34. Pisharath H, Rhee JM, Swanson M a, Leach SD, Parsons MJ (2007) Targeted ablation of beta cells in the embryonic zebrafish pancreas using E. coli nitroreductase. Mech Dev 124(3):218-29.

35. Ariga J, Walker SL, Mumm JS (2010) Multicolor time-lapse imaging of transgenic zebrafish: visualizing retinal stem cells activated by targeted neuronal cell ablation. $J$ Vis $\operatorname{Exp}(43): 2-7$.

36. Walker SL, et al. (2012) Automated reporter quantification in vivo: High-throughput screening method for reporter-based assays in zebrafish. PLoS One 7(1):e29916.

37. White DT, et al. (2016) ARQiv-HTS, a versatile whole-organism screening platform enabling in vivo drug discovery at high-throughput rates. Nat Protoc 11(12):2432-2453. 
38. Morris AC, Forbes-Osborne MA, Pillai LS, Fadool JM (2011) Microarray analysis of XOPS-mCFP zebrafish retina identifies genes associated with rod photoreceptor degeneration and regeneration. Investig Ophthalmol Vis Sci. doi:10.1167/iovs.10-6022.

39. Ariga J, Walker SL, Mumm JS (2010) Multicolor time-lapse imaging of transgenic zebrafish: Visualizing retinal stem cells activated by targeted neuronal cell ablation. $J$ Vis Exp. doi:10.3791/2093.

40. Schroeter EH, Wong ROL, Gregg RG (2006) In vivo development of retinal ON-bipolar cell axonal terminals visualized in nyx::MYFP transgenic zebrafish. Vis Neurosci 23(5):833-43.

41. Schmitt EA, Dowling JE (1996) Comparison of topographical patterns of ganglion and photoreceptor cell differentiation in the retina of the zebrafish, Danio rerio. $J$ Comp Neurol. doi:10.1002/(SICl)1096-9861(19960722)371:2<222::AID-CNE3>3.0.CO;2-4.

42. Larison KD, Bremiller R (1990) Early onset of phenotype and cell patterning in the embryonic zebrafish retina. Development.

43. Vihtelic TS, Doro CJ, Hyde DR (1999) Cloning and characterization of six zebrafish photoreceptor opsin cDNAs and immunolocalization of their corresponding proteins. Vis Neurosci. doi:10.1017/S0952523899163168.

44. Hamaoka T, Takechi M, Chinen A, Nishiwaki Y, Kawamura S (2002) Visualization of rod photoreceptor development using GFP-transgenic zebrafish. Genesis 34(3):215-20.

45. Gavrieli Y, Sherman Y, Ben-Sasson SA (1992) Identification of programmed cell death in situ via specific labeling of nuclear DNA fragmentation. J Cell Biol.

doi:10.1083/jcb.119.3.493.

46. Montgomery JE, Parsons MJ, Hyde DR (2010) A novel model of retinal ablation demonstrates that the extent of rod cell death regulates the origin of the regenerated zebrafish rod photoreceptors. J Comp Neurol 518(6):800-814.

47. Hochreiter S, Clevert DA, Obermayer K (2006) A new summarization method for affymetrix probe level data. Bioinformatics. doi:10.1093/bioinformatics/btl033.

48. Fausett B V., Gumerson JD, Goldman D (2008) The proneural basic helix-loop-helix gene Ascl1a is required for retina regeneration. $J$ Neurosci. doi:10.1523/JNEUROSCI.485307.2008.

49. Gorsuch RA, et al. (2017) Sox2 regulates Müller glia reprogramming and proliferation in the regenerating zebrafish retina via Lin28 and Ascl1a. Exp Eye Res. doi:10.1016/j.exer.2017.05.012.

50. Nelson CM, et al. (2012) Stat3 defines three populations of müller glia and is required for initiating maximal müller glia proliferation in the regenerating zebrafish retina. $J$ Comp Neurol. doi:10.1002/cne.23213.

51. Kassen SC, et al. (2007) Time course analysis of gene expression during light-induced photoreceptor cell death and regeneration in albino zebrafish. Dev Neurobiol. doi:10.1002/dneu.20362.

52. Mitchell DM, Sun C, Hunter SS, New DD, Stenkamp DL (2019) Regeneration associated 
transcriptional signature of retinal microglia and macrophages. Sci Rep. doi:10.1038/s41598-019-41298-8.

53. Sifuentes CJ, Kim JW, Swaroop A, Raymond PA (2016) Rapid, dynamic activation of Müller Glial stem cell responses in Zebrafish. Investig Ophthalmol Vis Sci 57(13):51485160.

54. Dryja TP, et al. (1990) A point mutation of the rhodopsin gene in one form of retinitis pigmentosa. Nature. doi:10.1038/343364a0.

55. Morris AC, Schroeter EH, Bilotta J, Wong ROL, Fadool JM (2005) Cone survival despite rod degeneration in XOPS-mCFP transgenic zebrafish. Investig Ophthalmol Vis Sci. doi:10.1167/iovs.05-0797.

56. Stearns G, Evangelista M, Fadool JM, Brockerhoff SE (2007) A mutation in the conespecific pde6 gene causes rapid cone photoreceptor degeneration in zebrafish. $J$ Neurosci. doi:10.1523/JNEUROSCI.3136-07.2007.

57. Qin Z, Raymond PA (2012) Microarray-based gene profiling analysis of müller gliaderived retinal stem cells in light-damaged retinas from adult zebrafish. Methods Mol Biol. doi:10.1007/978-1-61779-848-1_18.

58. Sturn A, Quackenbush J, Trajanoski Z (2002) Genesis: Cluster analysis of microarray data. Bioinformatics. doi:10.1093/bioinformatics/18.1.207.

59. Mitchell DM, Lovel AG, Stenkamp DL (2018) Dynamic changes in microglial and macrophage characteristics during degeneration and regeneration of the zebrafish retina. J Neuroinflammation. doi:10.1186/s12974-018-1185-6.

60. Palazzo I, Deistler K, Hoang T V., Blackshaw S, Fischer AJ (2020) NF-kB signaling regulates the formation of proliferating Müller glia-derived progenitor cells in the avian retina. Development. doi:10.1242/dev.183418.

61. Gallina D, Zelinka C, Fischer AJ (2014) Glucocorticoid receptors in the retina, Müller glia and the formation of Müller glia-derived progenitors. Dev. doi:10.1242/dev.109835.

62. Yamashita S, et al. (2002) Stat3 controls cell movements during zebrafish gastrulation. Dev Cell. doi:10.1016/S1534-5807(02)00126-0.

63. Avalle L, Pensa S, Regis G, Novelli F, Poli V (2012) STAT1 and STAT3 in tumorigenesis: A matter of balance. JAK-STAT. doi:10.4161/jkst.20045.

64. Hutchins AP, Diez D, Miranda-Saavedra D (2013) The IL-10/STAT3-mediated antiinflammatory response: Recent developments and future challenges. Brief Funct Genomics. doi:10.1093/bfgp/elt028.

65. Nakajima K, et al. (1996) A central role for Stat3 in IL-6-induced regulation of growth and differentiation in M1 leukemia cells. EMBO J. doi:10.1002/j.1460-2075.1996.tb00734.x.

66. Fukada T (1998) STAT3 orchestrates contradictory signals in cytokine-induced G1 to S cell-cycle transition. EMBO J. doi:10.1093/emboj/17.22.6670.

67. Carballo M, et al. (1999) Oxidative stress triggers STAT3 tyrosine phosphorylation and nuclear translocation in human lymphocytes. J Biol Chem. doi:10.1074/jbc.274.25.17580. 
68. Jorstad NL, et al. (2020) STAT Signaling Modifies Ascl1 Chromatin Binding and Limits Neural Regeneration from Muller Glia in Adult Mouse Retina. Cell Rep. doi:10.1016/j.celrep.2020.01.075.

69. Gao Y, Cimica V, Reich NC (2012) Suppressor of cytokine signaling 3 inhibits breast tumor kinase activation of STAT3. J Biol Chem. doi:10.1074/jbc.M111.334144.

70. Shodeinde AL, Barton BE (2012) Potential use of STAT3 inhibitors in targeted prostate cancer therapy: Future prospects. Onco Targets Ther. doi:10.2147/OTT.S32559.

71. Thanasaksiri K, Hirono I, Kondo H (2016) Identification and expression analysis of suppressors of cytokine signaling (SOCS) of Japanese flounder Paralichthys olivaceus. Fish Shellfish Immunol. doi:10.1016/j.fsi.2016.09.018.

72. Liang J, et al. (2012) The stat3/socs3a pathway is a key regulator of hair cell regeneration in zebrafish stat3/socs3a pathway: Regulator of hair cell regeneration. J Neurosci. doi:10.1523/JNEUROSCI.5785-10.2012.

73. Khaliq M, et al. (2018) Stat 3 regulates liver progenitor cell-driven liver regeneration in zebrafish. Gene Expr. doi:10.3727/105221618X15242506133273.

74. Liu KE, Frazier WA (2015) Phosphorylation of the BNIP3 C-terminus inhibits mitochondrial damage and cell death without blocking autophagy. PLoS One. doi:10.1371/journal.pone.0129667.

75. Call JA, Nichenko AS (2020) Autophagy: an essential but limited cellular process for timely skeletal muscle recovery from injury. Autophagy.

doi:10.1080/15548627.2020.1753000.

76. Campochiaro PA, Mir TA (2018) The mechanism of cone cell death in Retinitis Pigmentosa. Prog Retin Eye Res. doi:10.1016/j.preteyeres.2017.08.004.

77. Bigarella CL, Liang R, Ghaffari S (2014) Stem cells and the impact of ROS signaling. Dev. doi:10.1242/dev.107086.

78. Jeong EM, et al. (2018) Real-Time Monitoring of Glutathione in Living Cells Reveals that High Glutathione Levels Are Required to Maintain Stem Cell Function. Stem Cell Reports. doi:10.1016/j.stemcr.2017.12.007.

79. Lim J, et al. (2020) Glutathione dynamics determine the therapeutic efficacy of mesenchymal stem cells for graft-versus-host disease via CREB1-NRF2 pathway. Sci Adv. doi:10.1126/sciadv.aba1334.

80. Agathocleous M, et al. (2012) Metabolic differentiation in the embryonic retina. Nat Cell Biol. doi:10.1038/ncb2531.

81. Burgess RJ, Agathocleous M, Morrison SJ (2014) Metabolic regulation of stem cell function. $J$ Intern Med. doi:10.1111/joim.12247.

82. Hansson HA, Holmgren A, Norstedt G, Rozell B (1989) Changes in the distribution of insulin-like growth factor I, thioredoxin, thioredoxin reductase and ribonucleotide reductase during the development of the retina. Exp Eye Res. doi:10.1016/S00144835(89)80009-0. 
83. de La Rosa EJ, et al. (1994) Insulin and Insulin-like Growth Factor System Components Gene Expression in the Chicken Retina From Early Neurogenesis Until Late Development and Their Effect on Neuroepithelial Cells. Eur J Neurosci. doi:10.1111/j.1460-9568.1994.tb00573.x.

84. Hoang T (2019) Cross-species transcriptomic and epigenomic analysis reveals key regulators of injury response and neuronal regeneration in vertebrate retinas. Bioarxiv. doi:10.1017/CBO9781107415324.004.

85. Köster RW, Fraser SE (2001) Tracing transgene expression in living zebrafish embryos. Dev Biol 233(2):329-46.

86. Bech-Hansen NT, et al. (2000) Mutations in NYX, encoding the leucine-rich proteoglycan nyctalopin, cause X-linked complete congenital stationary night blindness. Nat Genet. doi:10.1038/81619.

87. Pusch CM, et al. (2000) The complete form of X-linked congenital stationary night blindness is caused by mutations in a gene encoding a leucine-rich repeat protein. Nat Genet. doi:10.1038/81627.

88. Davison JM, et al. (2007) Transactivation from Gal4-VP16 transgenic insertions for tissue-specific cell labeling and ablation in zebrafish. Dev Biol 304(2):811-24.

89. Godinho L, et al. (2005) Targeting of amacrine cell neurites to appropriate synaptic laminae in the developing zebrafish retina. Development 132(22):5069-79.

90. D'Agati G, et al. (2017) A defect in the mitochondrial protein Mpv17 underlies the transparent casper zebrafish. Dev Biol 430(1):11-17.

91. Ren JQ, McCarthy WR, Zhang H, Adolph AR, Li L (2002) Behavioral visual responses of wild-type and hypopigmented zebrafish. Vision Res 42(3):293-9.

92. Mumm JS, et al. (2006) In vivo imaging reveals dendritic targeting of laminated afferents by zebrafish retinal ganglion cells. Neuron 52(4):609-21.

93. Schroeder A, et al. (2006) The RIN: an RNA integrity number for assigning integrity values to RNA measurements. BMC Mol Biol 7(1):3.

94. Reimers M, Carey VJ (2006) [8] Bioconductor: An Open Source Framework for Bioinformatics and Computational Biology. Methods Enzymol. doi:10.1016/S00766879(06)11008-3.

95. Diboun I, Wernisch L, Orengo CA, Koltzenburg M (2006) Microarray analysis after RNA amplification can detect pronounced differences in gene expression using limma. BMC Genomics. doi:10.1186/1471-2164-7-252.

96. Rainer J, Sanchez-Cabo F, Stocker G, Sturn A, Trajanoski Z (2006) CARMAweb: Comprehensive R- and bioconductor-based web service for microarray data analysis. Nucleic Acids Res. doi:10.1093/nar/gkl038.

97. Freue GVC, et al. (2007) MDQC: A new quality assessment method for microarrays based on quality control reports. Bioinformatics. doi:10.1093/bioinformatics/btm487. 


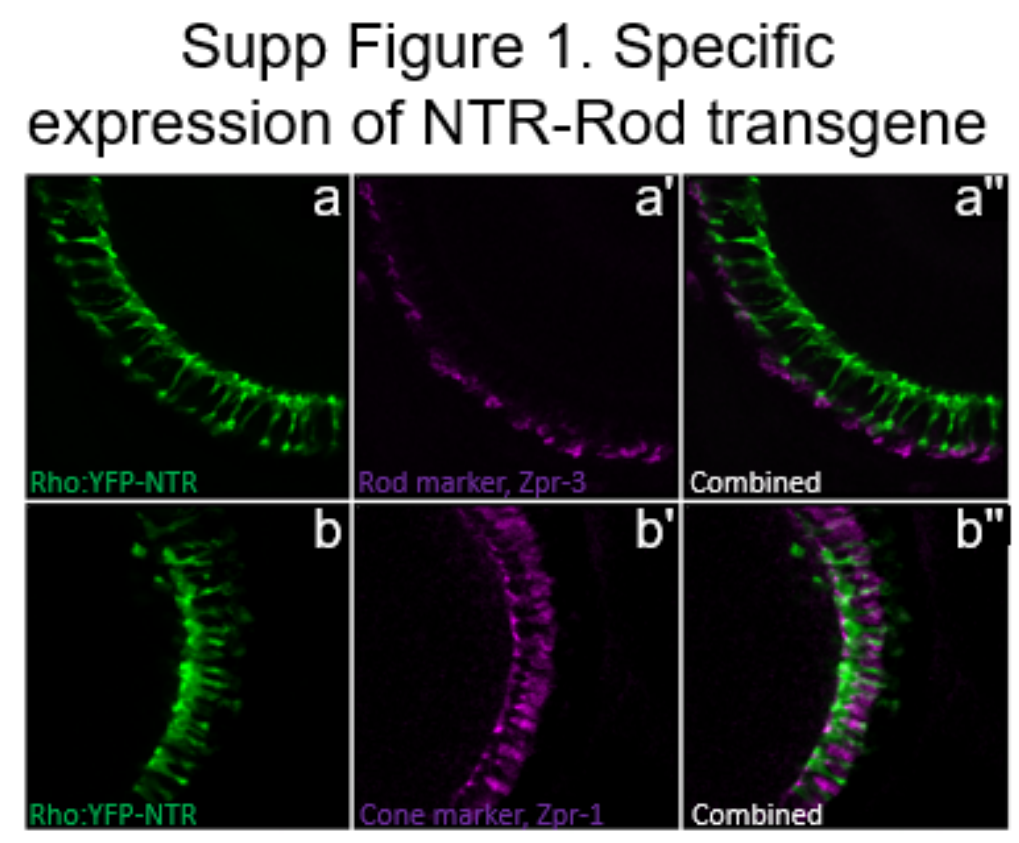

Supplementary Fig. 1. To test expression specificity of the gmc500 transgene, NTR rod larvae were sacrificed at $5 \mathrm{dpf}$, and eyes were cryosectioned at a thickness of $25 \mu \mathrm{m}$. Sections were processed for either zpr-3, a rod photoreceptor outer segment antibody (a-a"), or zpr-1, a redgreen double cone antibody immunoreactivity (b-b") and imaged using confocal microscopy. Rod outer segments labeled with zpr-3 (magenta) were directly apposed to YFP labeled NTR rod somata (yellow). D-F) Conversely, NTR rod cells (yellow) interdigitated between zpr-1 labeled cone photoreceptor somata (magenta). These data confirm that gmc500 transgene expression was restricted to rod photoreceptors. 
Figure 1. Assessment of NTRbipolar and NTR-rod ablation and regeneration
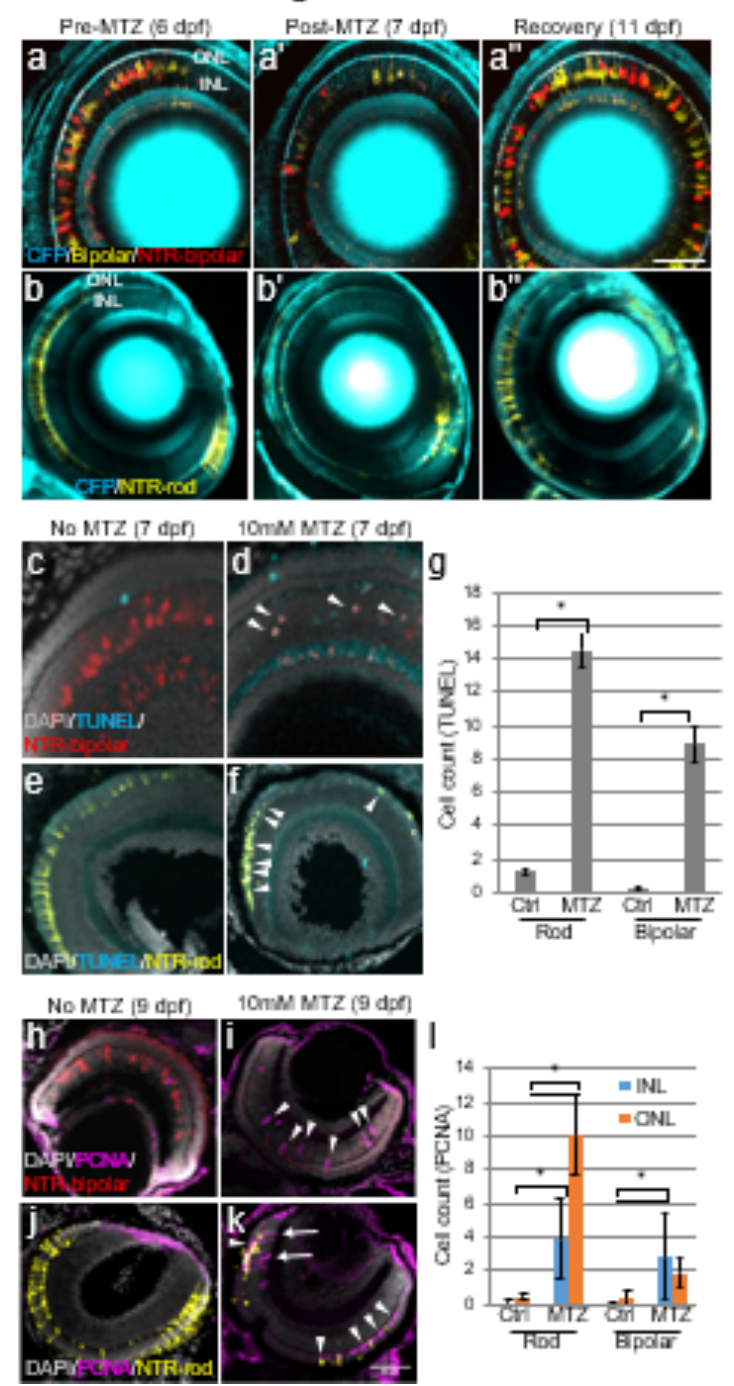

Figure 1: NTR-bipolar and NTR-rod zebrafish were ablated with $10 \mathrm{mM} \mathrm{MTZ}$ for $24 \mathrm{~h}$ at $6 \mathrm{dpf}$, resulting in rapid loss of transgene expression. By $11 \mathrm{dpf}$, robust regeneration of both lines was observed (a-a" for NTR-bipolar, b-b" for NTR-rod). Retinas were put through the TUNEL assay immediately following MTZ treatment to ensure selective cell death compared to untreated controls. MTZ treated retinas had numerous cells undergoing apoptosis in both lines (arrow heads, c-d for NTR-bipolar, e-f for NTR-rod, quantified in g). Next, PCNA labeling was performed $48 \mathrm{~h}$ into recovery to determine stem-cell response to retinal ablation. Untreated controls of the NTR-bipolar and NTR-rod zebrafish resulted in minimal proliferation in the retina ( $h$, j respectively). Following MTZ treatments in NTR-bipolar line, PCNA staining was observed throughout the retina in the INL and ONL while NTR-rod showed preferentially PCNA staining in the ONL (arrow heads, I, k respectively, quantified in I). Scale bar $=50$ microns; ${ }^{*} p<0.05$. 


\section{Supp Figure 2. Time series imaging of NTR-rod and NTR- bipolar ablation and regeneration}

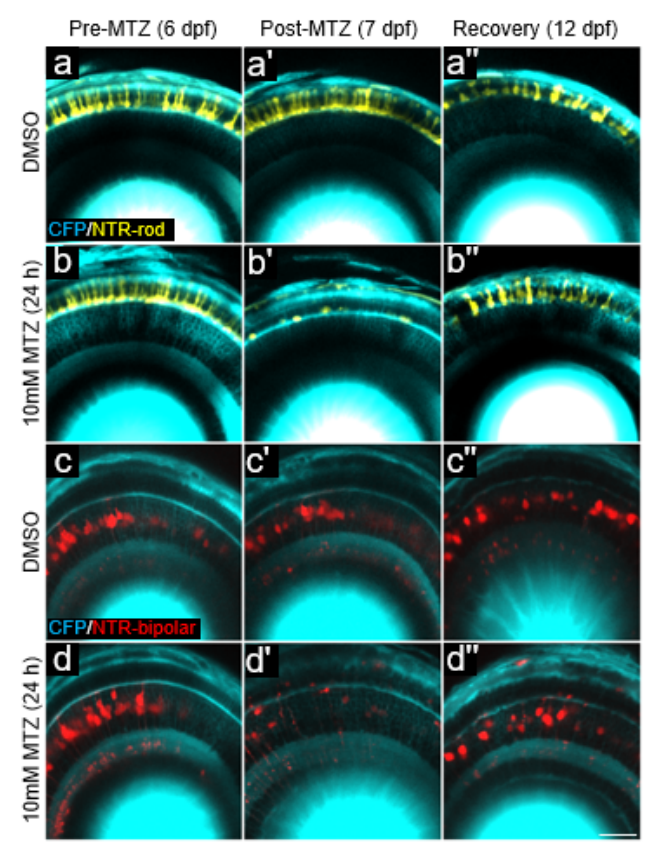

Supplemental Figure 2. Time series imaging was used to compare pre- and post-MTZ treated larvae. When NTR-rod lines were treated with DMSO (control), no substantial loss of photoreceptors was observed (Supp Fig. 2a, a'). Conversely, upon treatment with 10mM MTZ for $24 \mathrm{hr}$ (6-7 dpf) a rapid loss of photoreceptors occurred (Supp Fig. 2b, b'). Subsequent wash out and recovery over the next 5 days (12 dpf) revealed a robust regenerative response to replenish lost photoreceptors (Supp Fig. 3b"). Similar treatment of NTR-bipolar lines produced equivalent results; DMSO-treated controls resulted in no loss of bipolar interneurons (Supp Fig. 2c-c") while $24 \mathrm{hr}$ MTZ treatment (6-7 dpf) and washout led to rapid bipolar cell loss and subsequent regeneration, respectively (Supp Fig. 2d-d"). Scale bar $=50$ microns. 


\section{Figure 2. Microarray Analysis of Two Cell-specific Regeneration Paradigms}

\section{a}

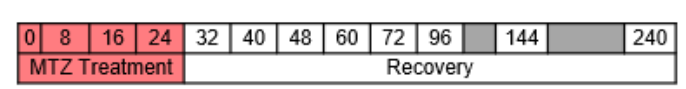

b

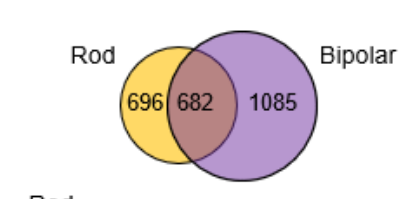

C

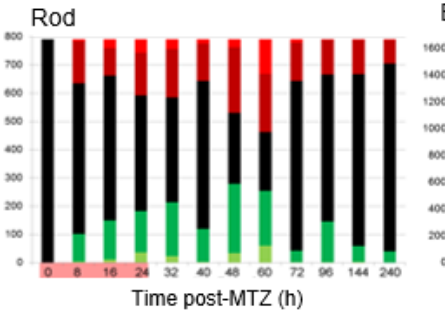

d

$\mathrm{d}_{\text {Rod } \mathrm{h} \text { post-MTZ }}$

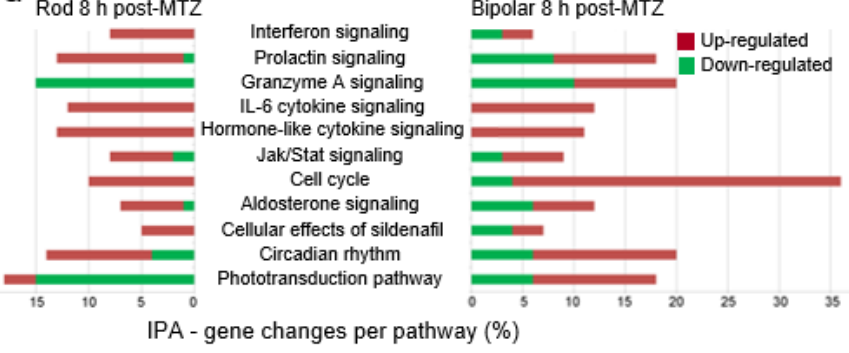

Figure 2. Gene expression similarities and differences were analyzed over a 10 day time course encompassing the loss and regeneration of either NTR-rod or NTR-bipolar cells. Starting at 0 hours, relative to initial exposure to MTZ at $6 \mathrm{dpf}, 12$ microarrays were collected for each condition in triplicate along with control retinas (a). With a 1.5 -fold change in expression cutoff and $p$-value $<=0.05,696$ and 1085 genes were differentially expressed in the rod and bipolar regeneration paradigms, respectively. 682 genes were shared between the two paradigms (b). A summary of differentially up regulated (red) and down regulated (green) genes at each time point for each paradigm (c). To elucidate differences between the paradigms, we used Ingenuity software to cluster genes at $8 \mathrm{hpt}$ into different genetic pathways. Similarities were observed in some genetic pathways, e.g. Jak/stat signaling and circadian rhythm; differences were observed in others, e.g. interferon signaling and granzyme A signaling (d). 


\section{Supp Table 1. mRNA quality of replicates used in microarray expression analysis.}

\begin{tabular}{cccc}
\hline Run & Cell Type & $\begin{array}{c}\text { Concentration } \\
(\mathbf{n g})\end{array}$ & mRNA Quality (RIN) \\
\hline $\mathbf{1}$ & Rod & $128.8-2327$ & $6.6-10$ \\
$\mathbf{2}$ & Rod & $109.2-992$ & $7.8-9.8$ \\
$\mathbf{3}$ & Rod & $136.0-1728$ & $9.8-10$ \\
\hline $\mathbf{4}$ & Bipolar & $141.0-1029$ & $5.9-9.4$ \\
$\mathbf{5}$ & Bipolar & $145.4-990.8$ & $8.2-9.3$ \\
\hline $\mathbf{6}$ & Bipolar & $204.5-685.4$ & $8.4-9.4$ \\
\hline
\end{tabular}

Supplemental Table 1: RNA sample quality was assessed by concentration and the RNA Integrity Number (RIN), an algorithm that assigns integrity values to RNA measurements. Only samples attaining a minimal concentration of $\geq 100 \mathrm{ng} / \mu \mathrm{L}(5 \mu \mathrm{L}$ total) and a RNA integrity number (RIN) value of $\geq 8$ were used for microarray hybridization. Runs where a minimum of 21 of 23 samples were deemed of good quality were utilized in the microarray studies. Three runs were processed per regenerative paradigm resulting in six microarray sets and a total 138 microarrays overall. 

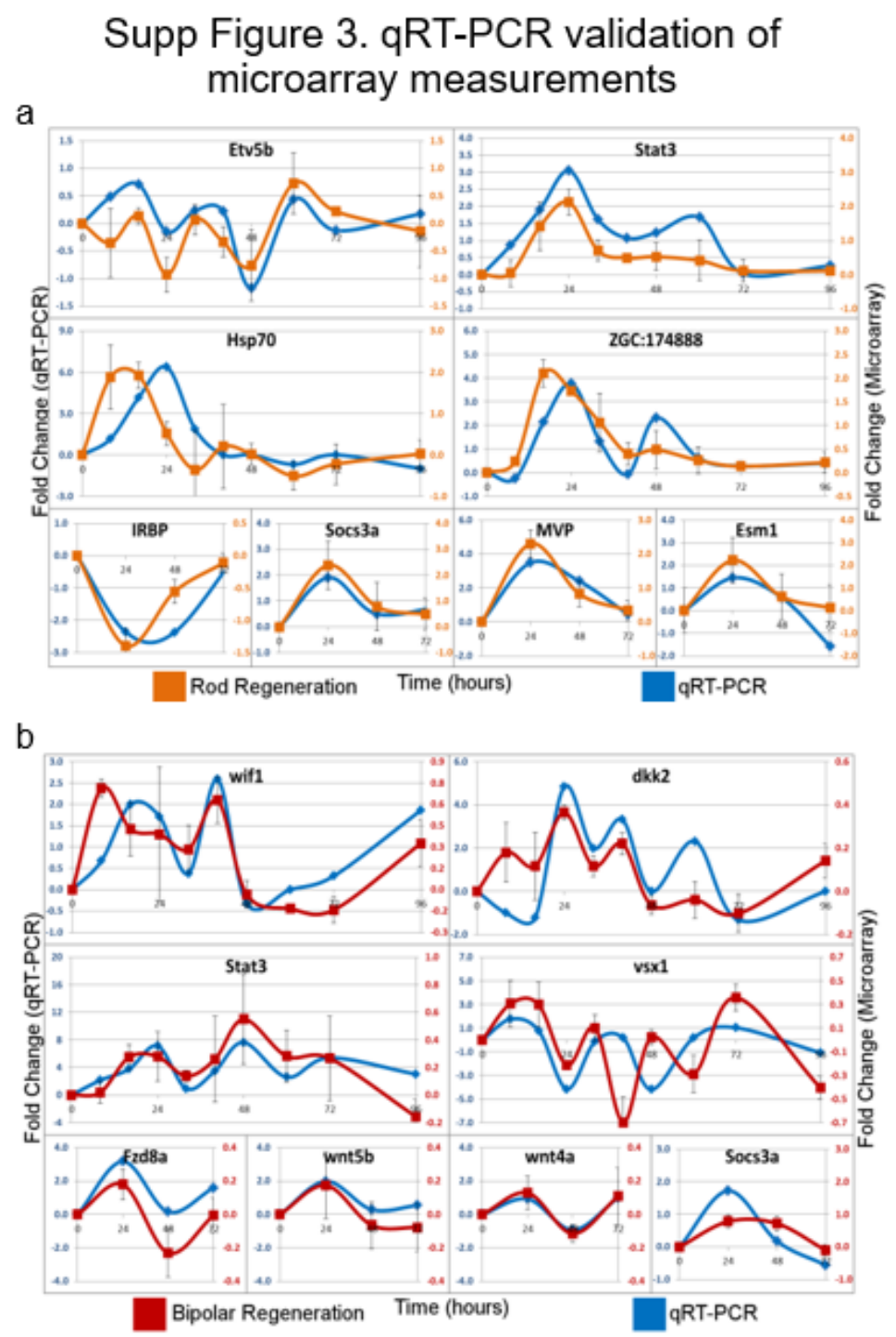

Supplemental Figure 3. qRT-PCR was performed with residual mRNA from microarray experiments to validate select genes in both regenerative paradigms. Samples were run in triplicate per plate at 50 cycles to ensure fold changes were calculated accurately. All PCR samples were normalized to beta-actin to ensure equal loading per time point. Due to separate normalization steps for qRT-PCR and microarray, microarray data was up-scaled by factors of $2 \mathrm{x}, 3 \mathrm{x}$, and 10x to simplify comparisons of gene expression trends (right scale bar) to the qRT-PCR fold changes (left scale bar). Rod qRT-PCR A) qRT-PCR validated genes from the $j a k / s t a t$ pathway from the rod regeneration microarray data, with no significant differences occurring temporally between the two (A). Bipolar qRT-PCR B) Similar results were observed when comparing bipolar regeneration microarray data to qRT-PCR, with select wnt and jak-stat pathway genes showing no temporal differences (B). 


\section{Supp Fig 4. NTR-rod/bipolar expression data, 8h post-MTZ}
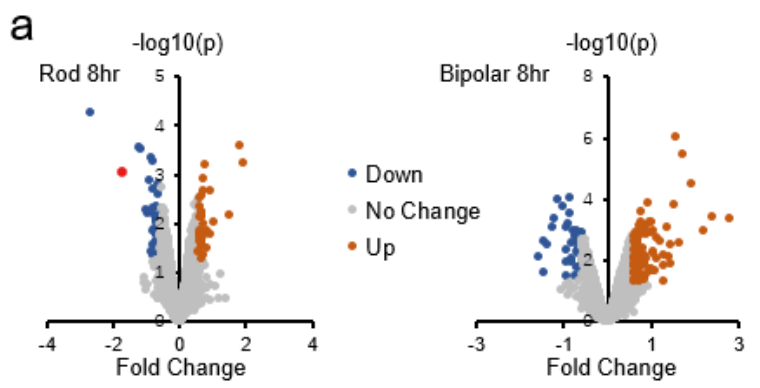

b
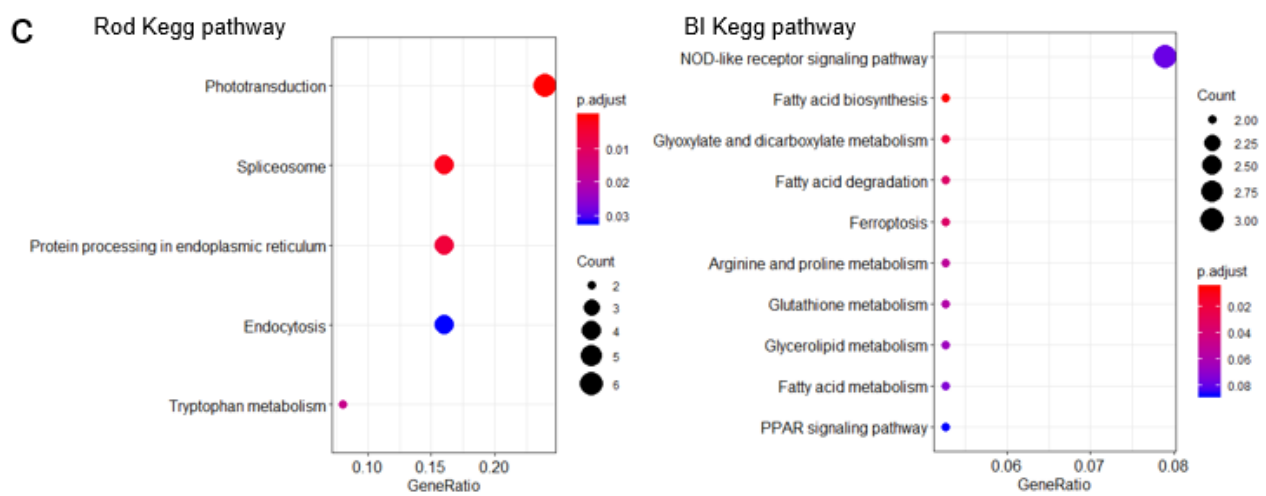

Supplemental Figure 4. RNA expression changes at 8 hours post-Mtz ablation. Volcano parts depicting gene expression changes as function of fold change in Rod and Bipolar cells (a). Number of shared differentially expressed genes in Rods (yellow) and Bipolar cells (green), shared genes in the middle (b). Lastly, differentially expressed genes were classified by known pathway involvement using KEGG analysis. Circles shown indicate statistically significant changes in adjusted p-value (c). 


\section{Supp Fig 5. NTR-rod/bipolar expression data, 16h post-MTZ}
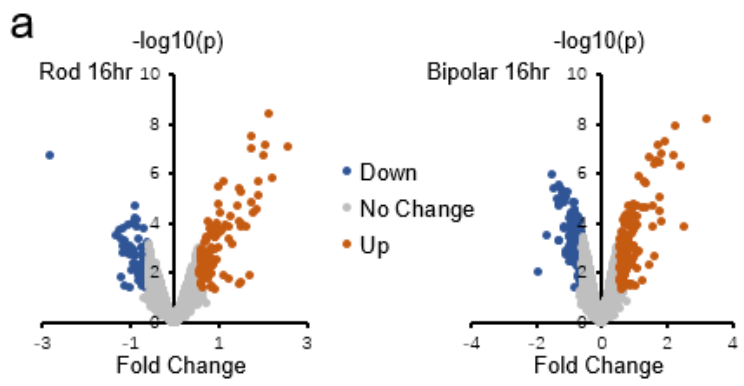

b
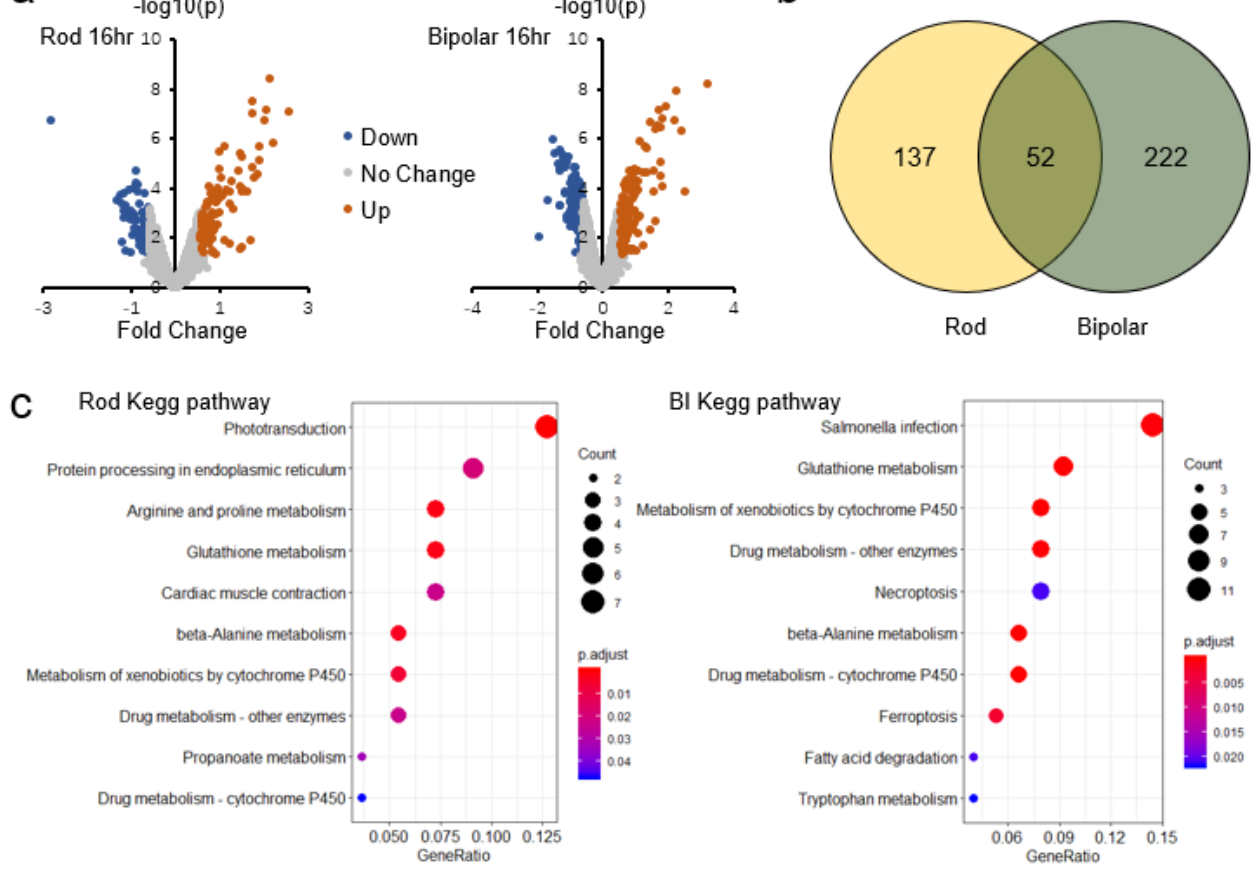

Supplemental Figure 5. RNA expression changes at 16 hours post-Mtz ablation. Volcano parts depicting gene expression changes as function of fold change in Rod and Bipolar cells (a). Number of shared differentially expressed genes in Rods (yellow) and Bipolar cells (green), shared genes in the middle (b). Lastly, differentially expressed genes were classified by known pathway involvement using KEGG analysis. Circles shown indicate statistically significant changes in adjusted p-value (c). 


\section{Supp Fig 6. NTR-rod/bipolar expression data, 24h post-MTZ}

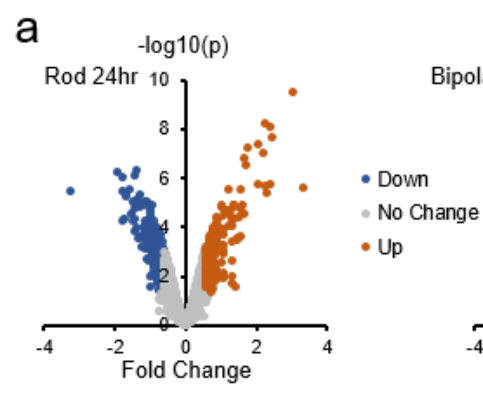

$-\log 10(p)$

$\mathrm{b}$
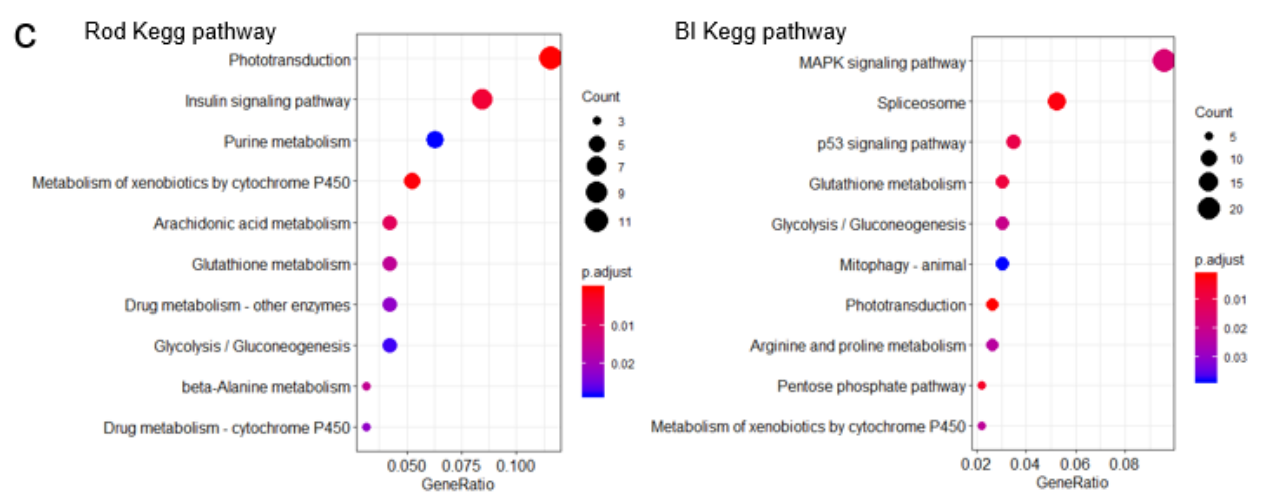

Supplemental Figure 6. RNA expression changes at 24 hours post-Mtz ablation. Volcano parts depicting gene expression changes as function of fold change in Rod and Bipolar cells (a). Number of shared differentially expressed genes in Rods (yellow) and Bipolar cells (green), shared genes in the middle (b). Lastly, differentially expressed genes were classified by known pathway involvement using KEGG analysis. Circles shown indicate statistically significant changes in adjusted p-value (c). 


\section{Supp Fig 7. NTR-rod/bipolar expression data, 32h post-MTZ}
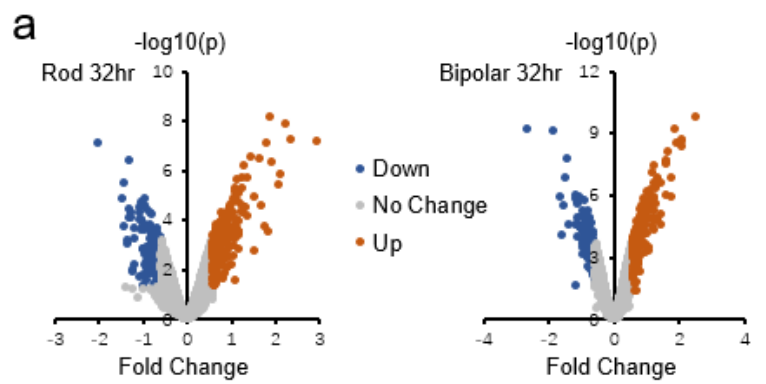

b
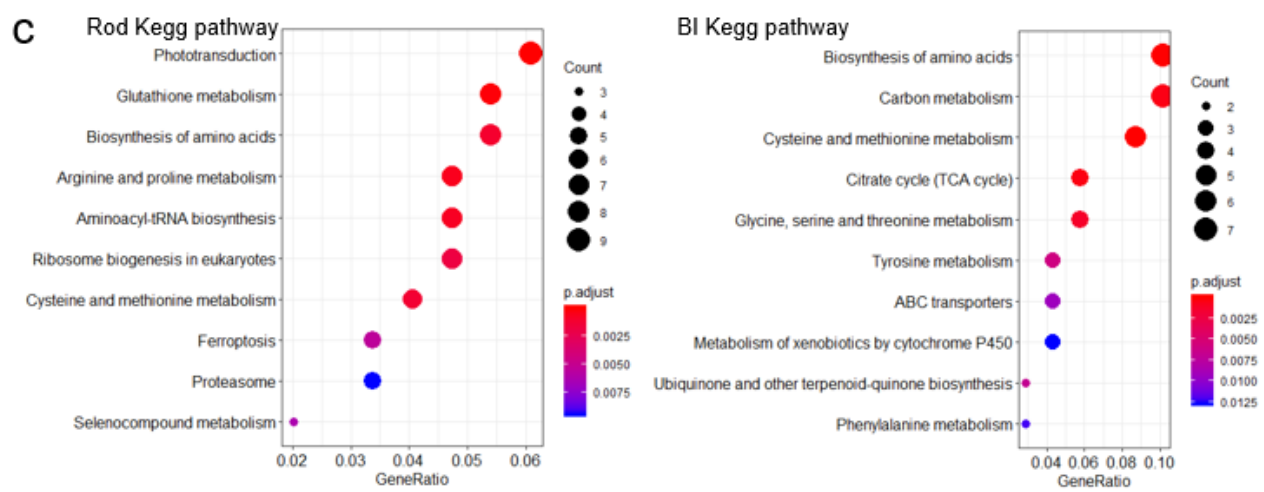

Supplemental Figure 7. RNA expression changes at 32 hours post-Mtz ablation. Volcano parts depicting gene expression changes as function of fold change in Rod and Bipolar cells (a). Number of shared differentially expressed genes in Rods (yellow) and Bipolar cells (green), shared genes in the middle (b). Lastly, differentially expressed genes were classified by known pathway involvement using KEGG analysis. Circles shown indicate statistically significant changes in adjusted p-value (c). 


\section{Supp Fig 8. NTR-rod/bipolar expression data, 40h post-MTZ}

a

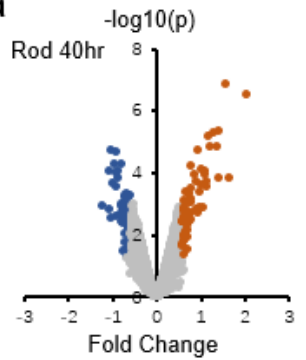

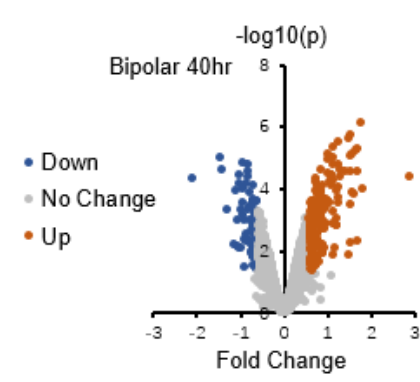

b

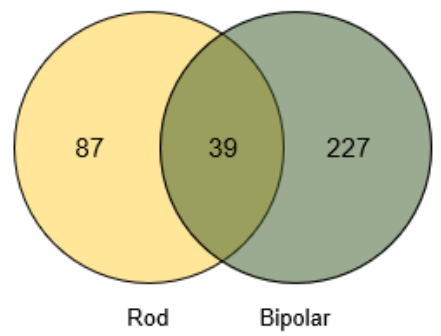

C

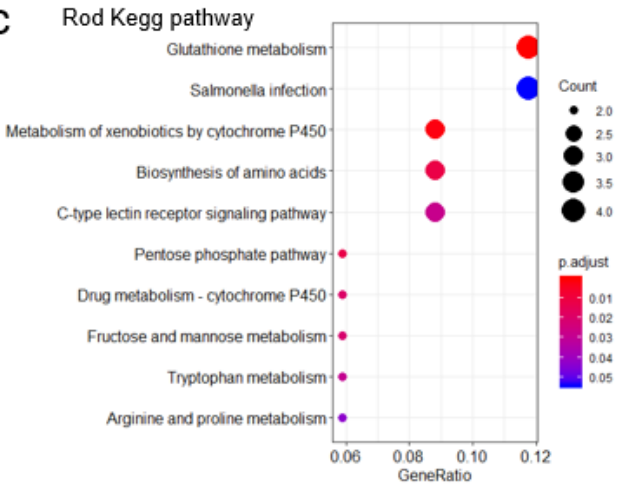

BI Kegg pathway

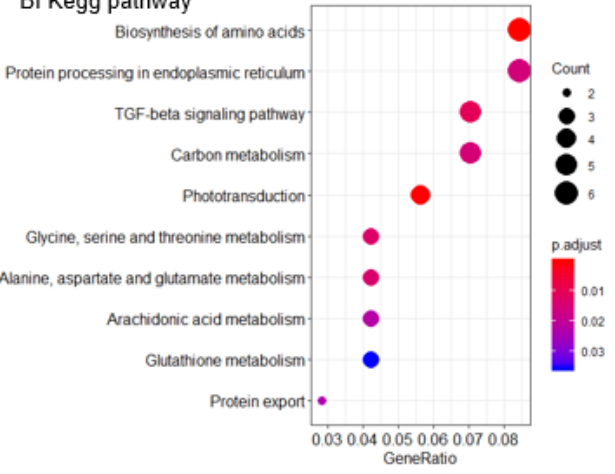

Supplemental Figure 8. RNA expression changes at 40 hours post-Mtz ablation. Volcano parts depicting gene expression changes as function of fold change in Rod and Bipolar cells (a). Number of shared differentially expressed genes in Rods (yellow) and Bipolar cells (green), shared genes in the middle (b). Lastly, differentially expressed genes were classified by known pathway involvement using KEGG analysis. Circles shown indicate statistically significant changes in adjusted p-value (c). 


\section{Supp Fig 9. NTR-rod/bipolar expression data, 48h post-MTZ}

a

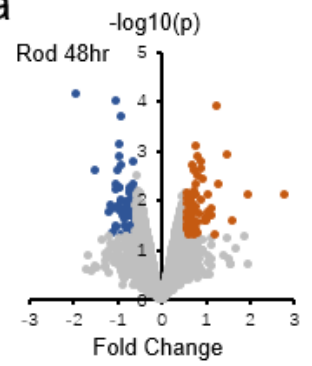

b
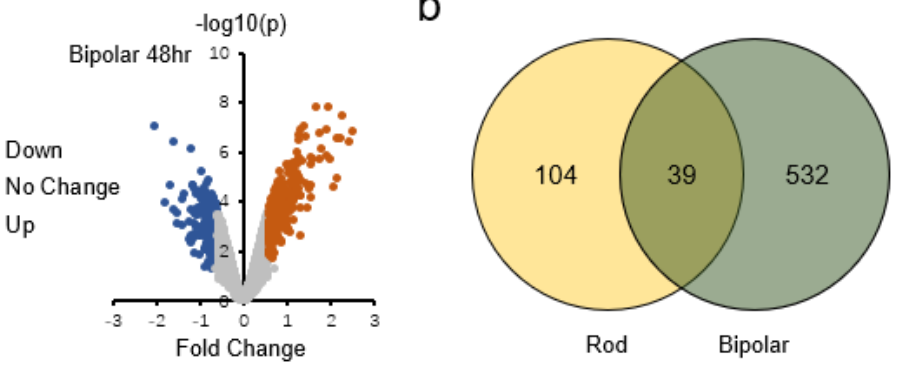

C
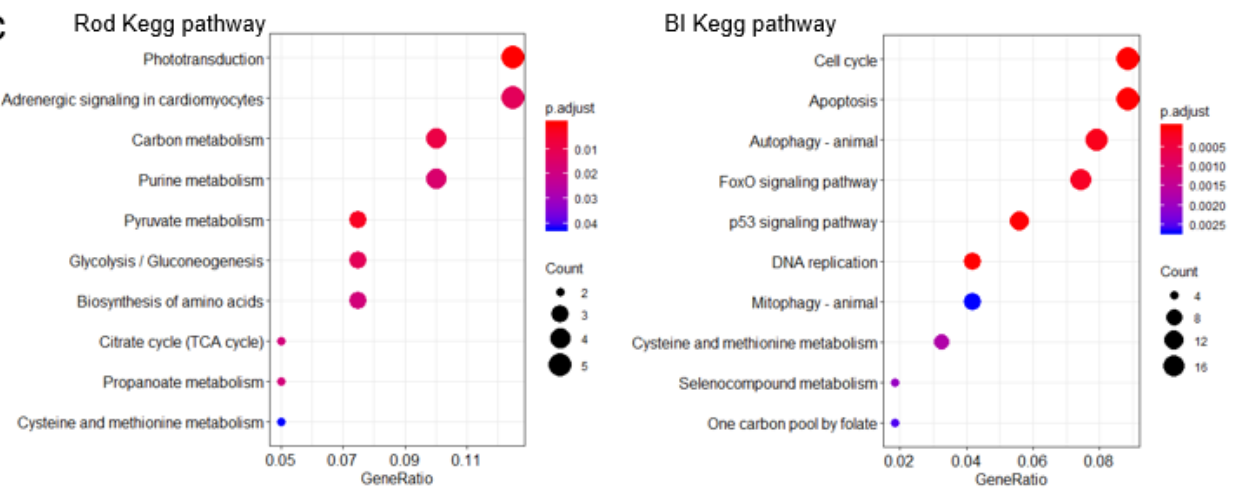

Supplemental Figure 9. RNA expression changes at 48 hours post-Mtz ablation. Volcano parts depicting gene expression changes as function of fold change in Rod and Bipolar cells (a). Number of shared differentially expressed genes in Rods (yellow) and Bipolar cells (green), shared genes in the middle (b). Lastly, differentially expressed genes were classified by known pathway involvement using KEGG analysis. Circles shown indicate statistically significant changes in adjusted p-value (c). 


\section{Supp Fig 10. NTR-rod/bipolar expression data, 60h post-MTZ}

a
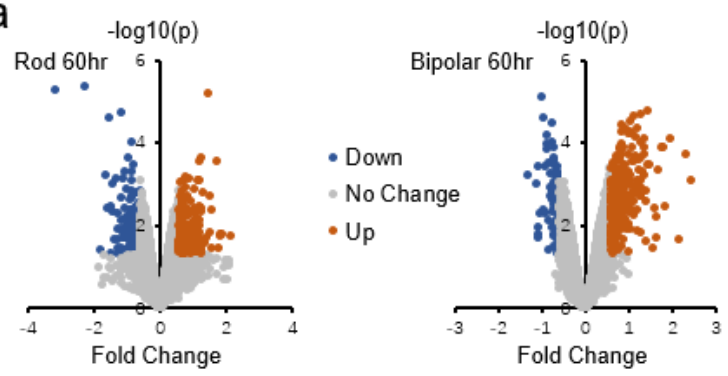

C

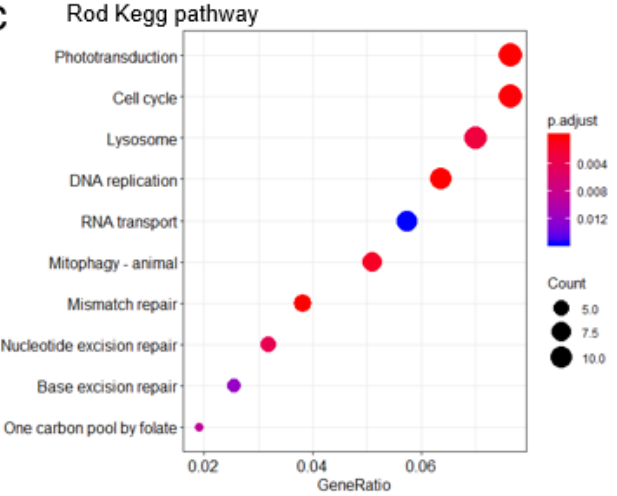

b

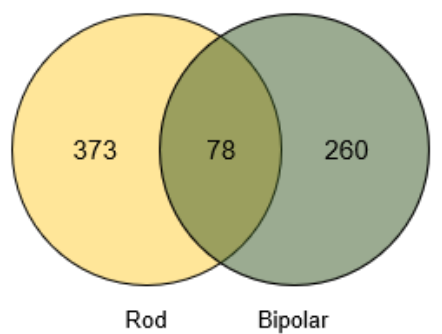

BI Kegg pathway

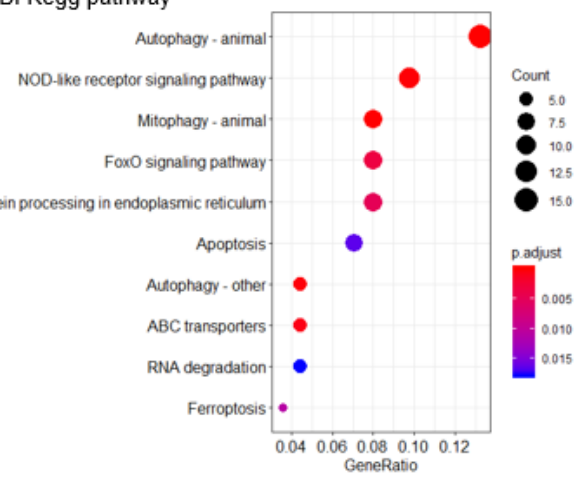

Supplemental Figure 10. RNA expression changes at 60 hours post-Mtz ablation. Volcano parts depicting gene expression changes as function of fold change in Rod and Bipolar cells (a). Number of shared differentially expressed genes in Rods (yellow) and Bipolar cells (green), shared genes in the middle (b). Lastly, differentially expressed genes were classified by known pathway involvement using KEGG analysis. Circles shown indicate statistically significant changes in adjusted p-value (c). 


\section{Supp Fig 11. NTR-rod/bipolar expression data, 72h post-MTZ}
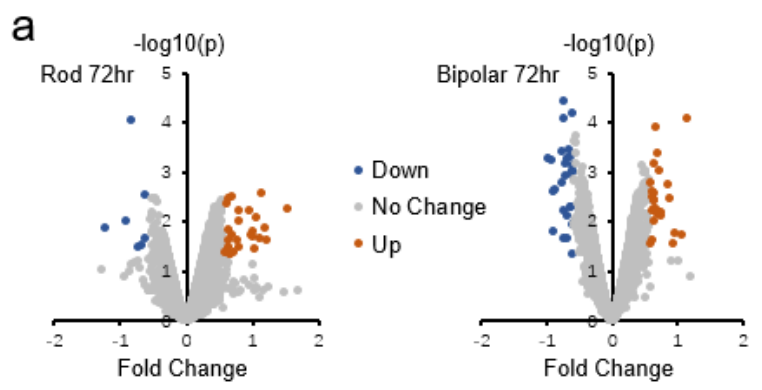

b
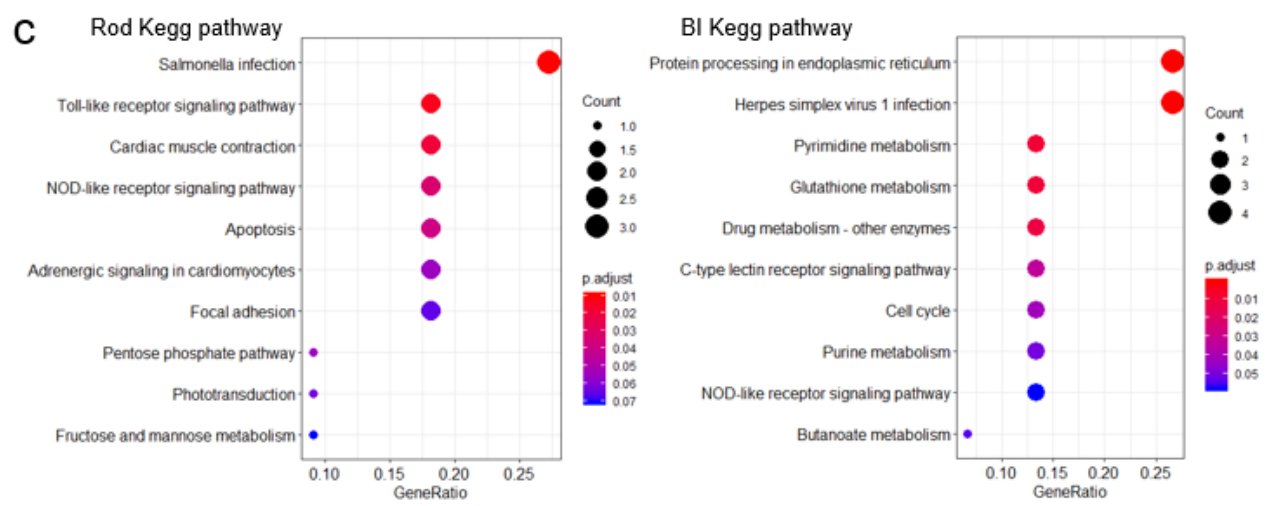

Supplemental Figure 11. RNA expression changes at 72 hours post-Mtz ablation. Volcano parts depicting gene expression changes as function of fold change in Rod and Bipolar cells (a). Number of shared differentially expressed genes in Rods (yellow) and Bipolar cells (green), shared genes in the middle (b). Lastly, differentially expressed genes were classified by known pathway involvement using KEGG analysis. Circles shown indicate statistically significant changes in adjusted p-value (c). 


\section{Supp Fig 12. NTR-rod/bipolar expression data, 96h post-MTZ}
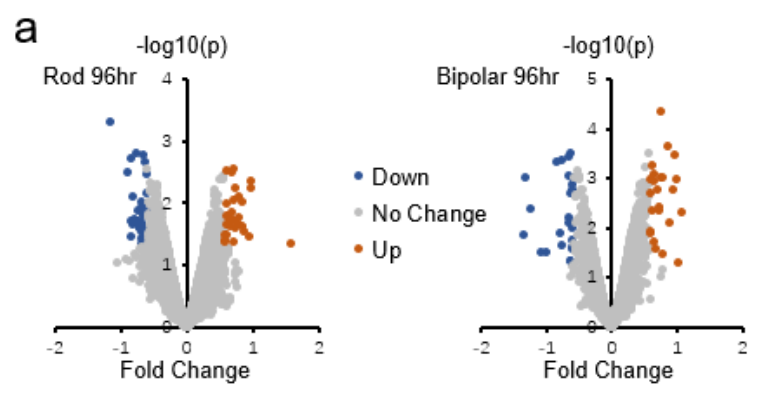

$\mathrm{b}$
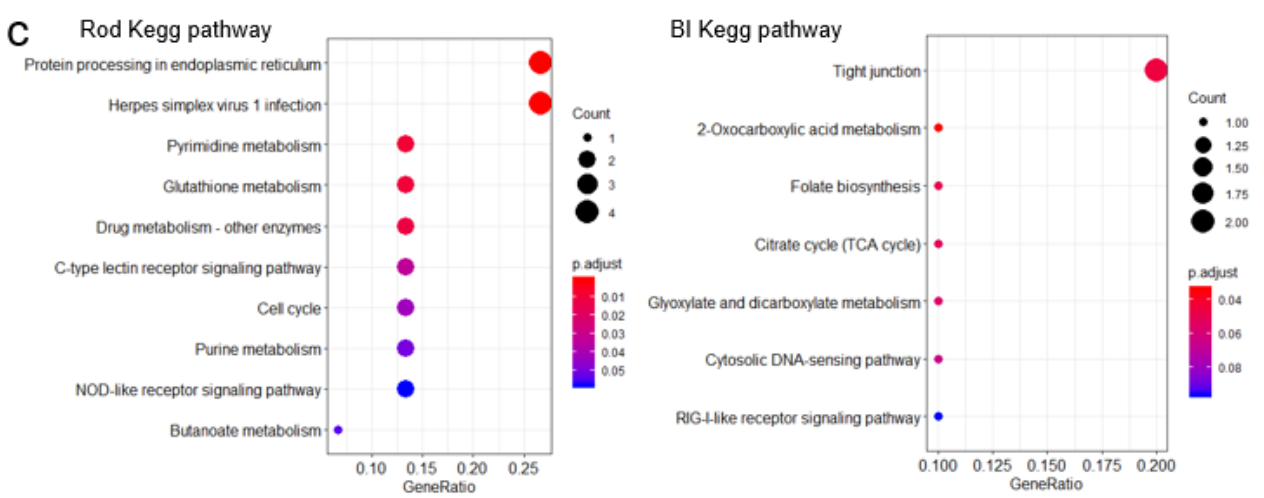

Supplemental Figure 12. RNA expression changes at 96 hours post-Mtz ablation. Volcano parts depicting gene expression changes as function of fold change in Rod and Bipolar cells (a). Number of shared differentially expressed genes in Rods (yellow) and Bipolar cells (green), shared genes in the middle (b). Lastly, differentially expressed genes were classified by known pathway involvement using KEGG analysis. Circles shown indicate statistically significant changes in adjusted p-value (c). 


\section{Supp Fig 13. NTR-rod/bipolar expression data, 144h post-MTZ}
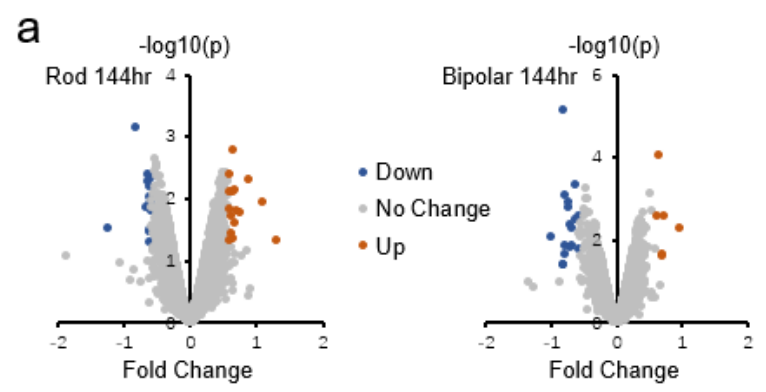

b
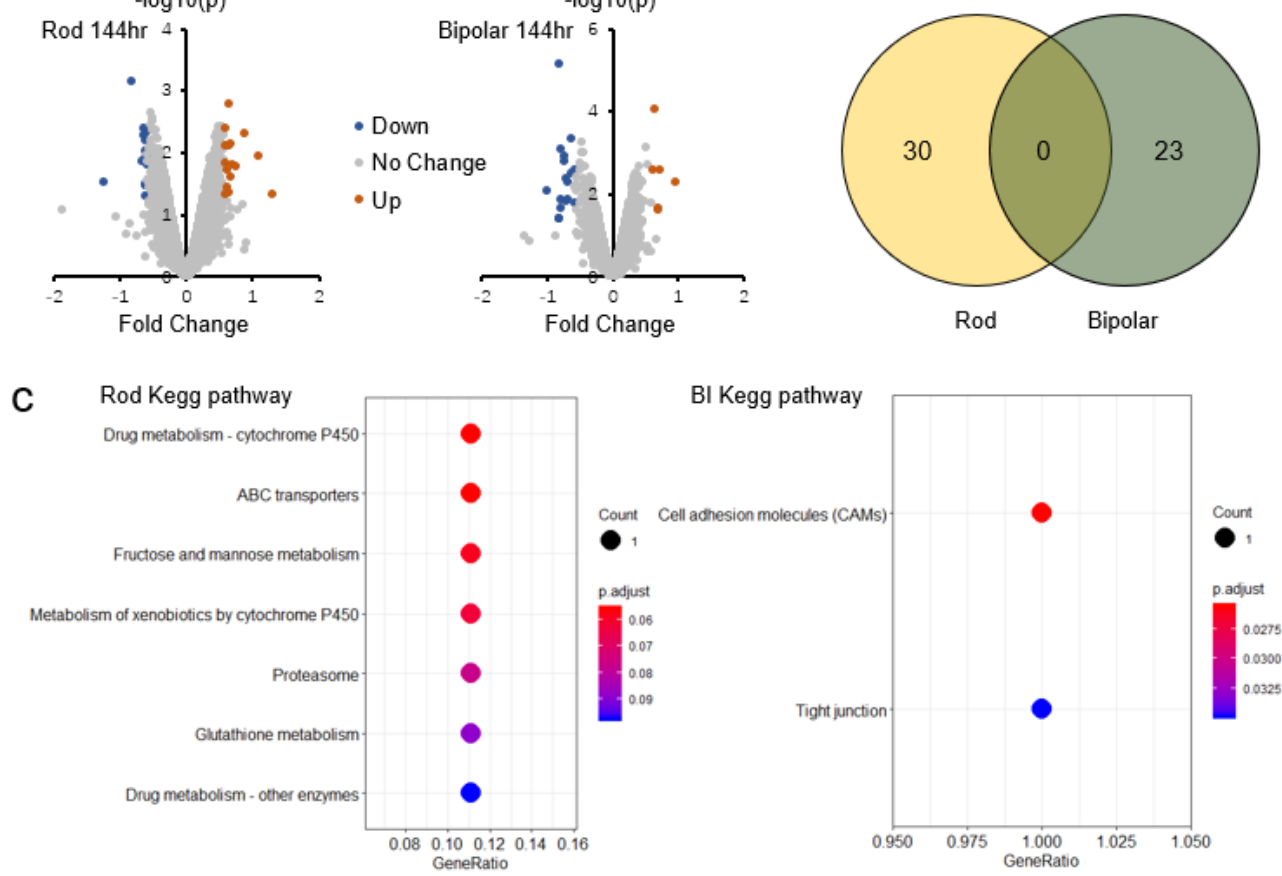

Supplemental Figure 13. RNA expression changes at 144 hours post-Mtz ablation. Volcano parts depicting gene expression changes as function of fold change in Rod and Bipolar cells (a). Number of shared differentially expressed genes in Rods (yellow) and Bipolar cells (green), shared genes in the middle (b). Lastly, differentially expressed genes were classified by known pathway involvement using KEGG analysis. Circles shown indicate statistically significant changes in adjusted p-value (c). 


\section{Supp Fig 14. NTR-rod/bipolar expression data, 240h post-MTZ}

a
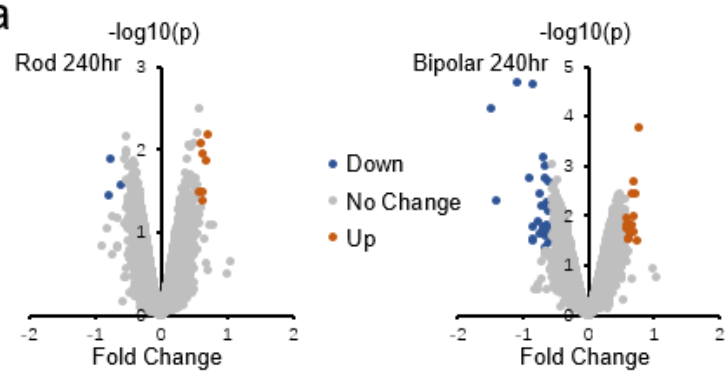

b

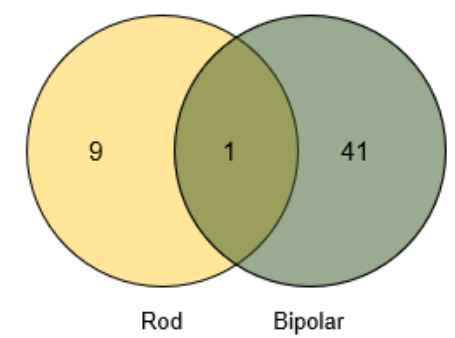

C
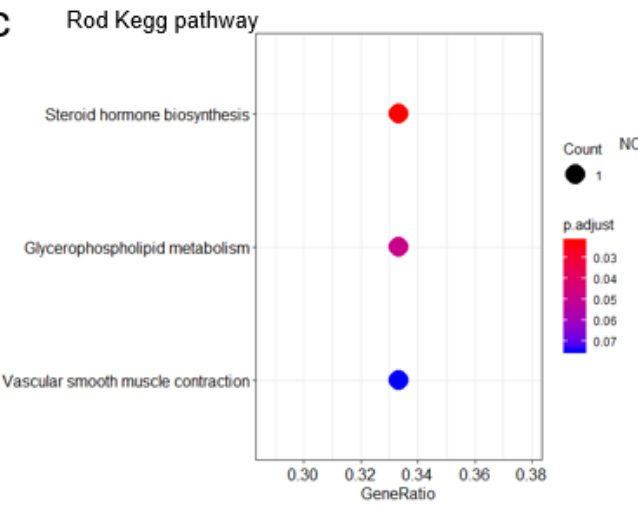

BI Kegg pathway

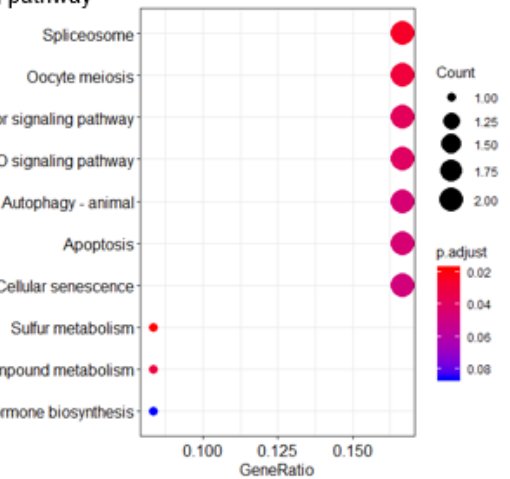

Supplemental Figure 14. RNA expression changes at 240 hours post-Mtz ablation. Volcano parts depicting gene expression changes as function of fold change in Rod and Bipolar cells (a). Number of shared differentially expressed genes in Rods (yellow) and Bipolar cells (green), shared genes in the middle (b). Lastly, differentially expressed genes were classified by known pathway involvement using KEGG analysis. Circles shown indicate statistically significant changes in adjusted p-value (c). 


\section{Figure 3. Dynamic gene expression patterns emphasized at early or late regenerative timepoints}
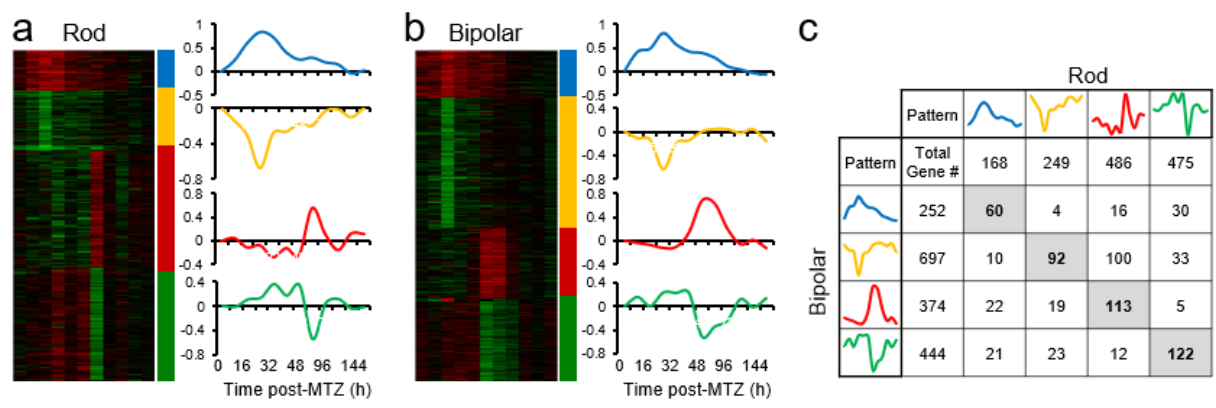

Figure 3. Using KEGG pathway analysis, dynamically regulated pathways (up and down) were identified in both NTR-rod and NTR-bipolar cell ablation paradigms. Early timepoints were defined as 24 and 32 hours after initial MTZ treatment and late timepoints as 60 and 72 hours. This yielded 4 patterns (early + up, early + down, late + up, and late + down). Comparison of these pathways identified commonly regulated genes between each paradigm. In total, 387 common genes (differentially regulated in the same way at the same timepoint) were observed, with a larger proportion favoring the later timepoints (c). 


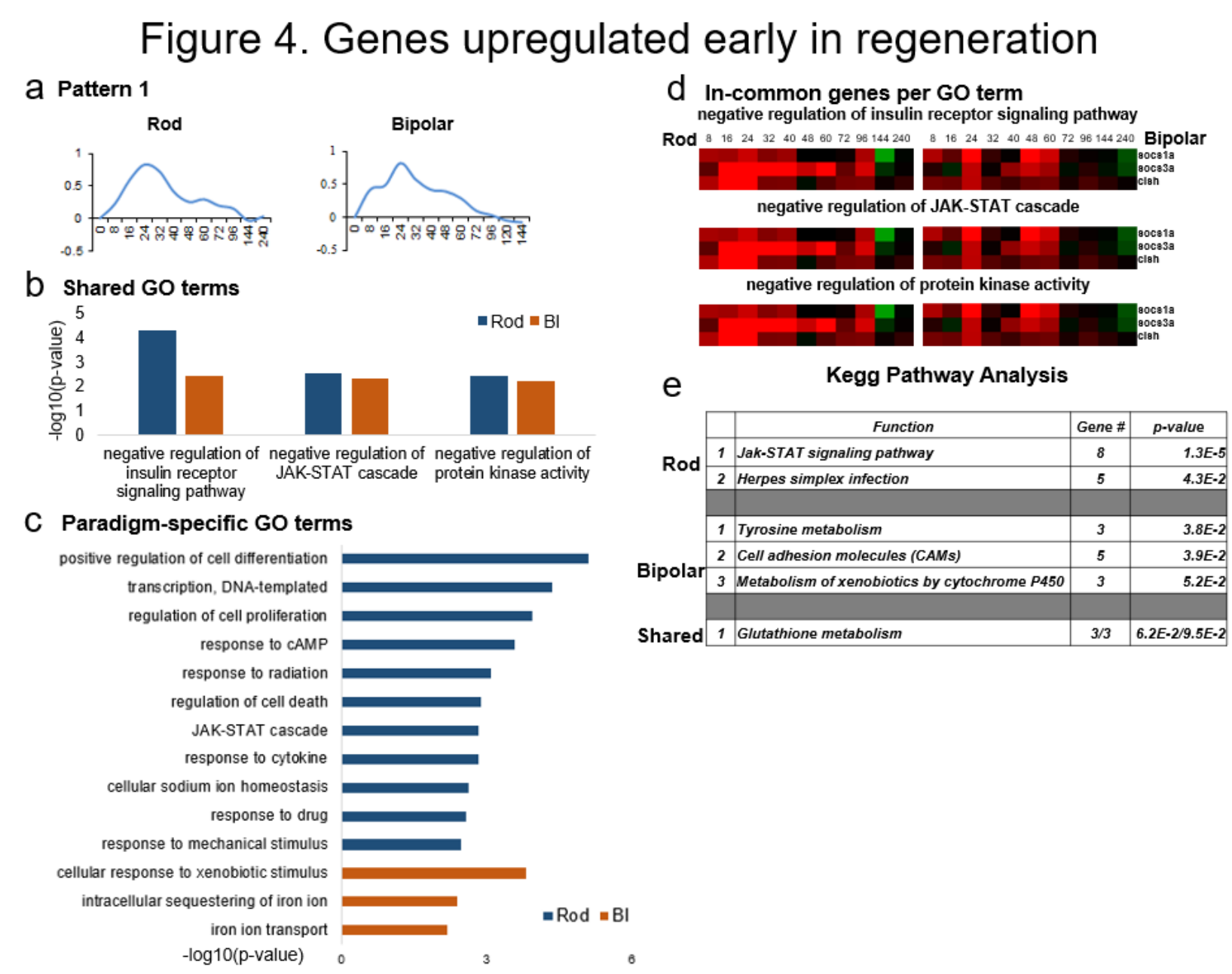

Figure 4: Analysis of Pattern 1; genes upregulated in early regeneration in Rod and Bipolar paradigms. Graphs corresponding to relative expression over the full experiment duration of genes upregulated early (2-fold difference compared to control) in regeneration in both paradigms (a). Gene ontology (GO) was used to identify shared pathway regulatory changes between Rod PRs (Rod) and Bipolar (BI) cells (b) and then paradigm-specific changes $(c)$, listed terms reached shared significance $(\mathrm{p}<0.05)$. Then, GO was further broken down into commonly differentially regulated genes and associated GO terms (d). Lastly, KEGG pathway analysis was used to find paradigm-specific and shared pathway changes that reached significance $(p<0.05)(e)$. 


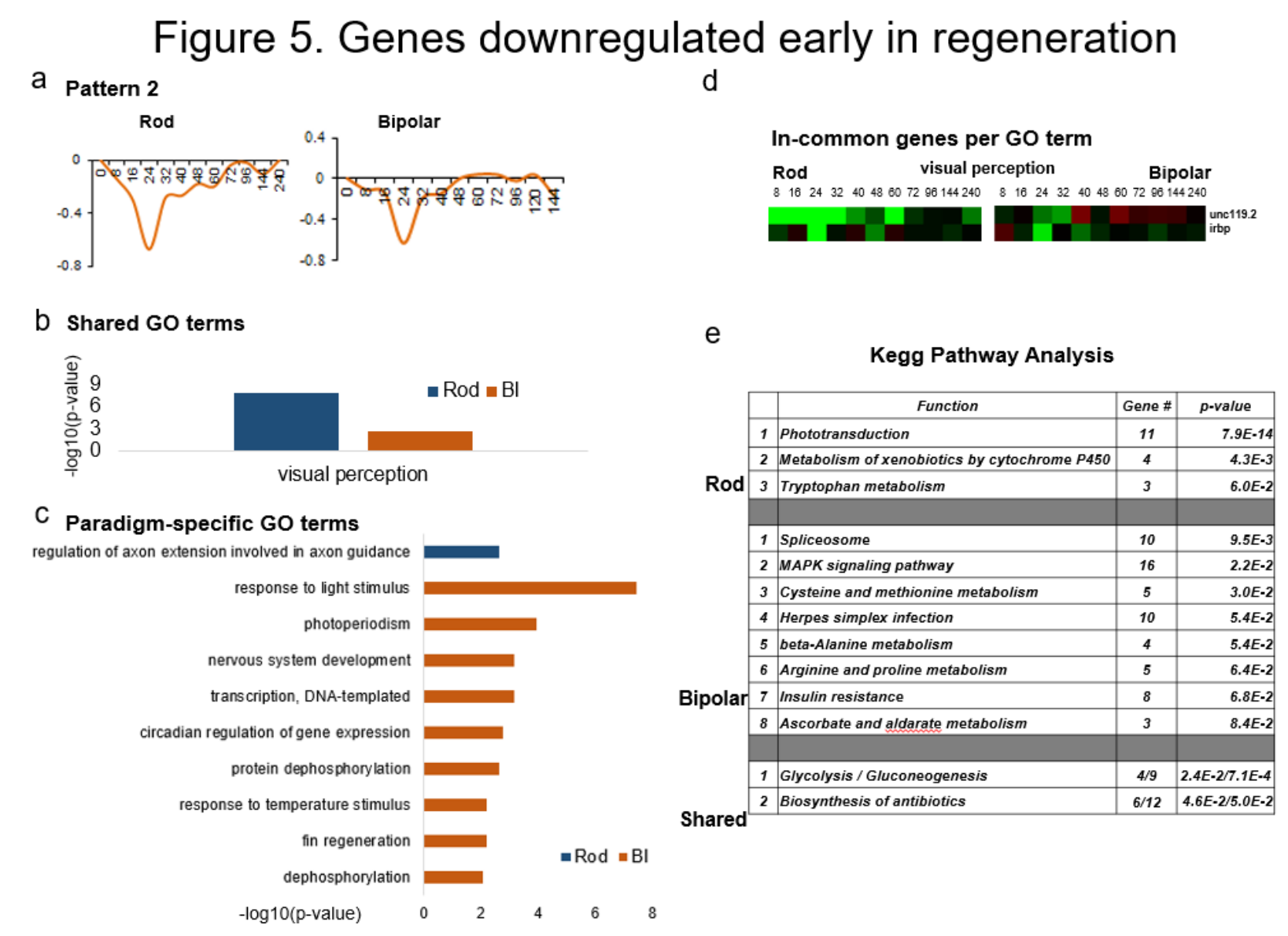

Figure 5: Analysis of Pattern 2; genes downregulated in early regeneration in Rod and Bipolar paradigms. Graphs corresponding to relative expression over the full experiment duration of genes downregulated early (2-fold difference compared to control) in regeneration in both paradigms (a). Gene ontology (GO) was used to identify shared pathway regulatory changes between Rod PRs (Rod) and Bipolar (BI) cells (b) and then paradigm-specific changes (c), listed terms reached shared significance $(\mathrm{p}<0.05)$. Then, GO was further broken down into commonly differentially regulated genes and associated GO terms (d). Lastly, KEGG pathway analysis was used to find paradigm-specific and shared pathway changes that reached significance $(\mathrm{p}<0.05)(\mathrm{e})$. 
a Pattern 3
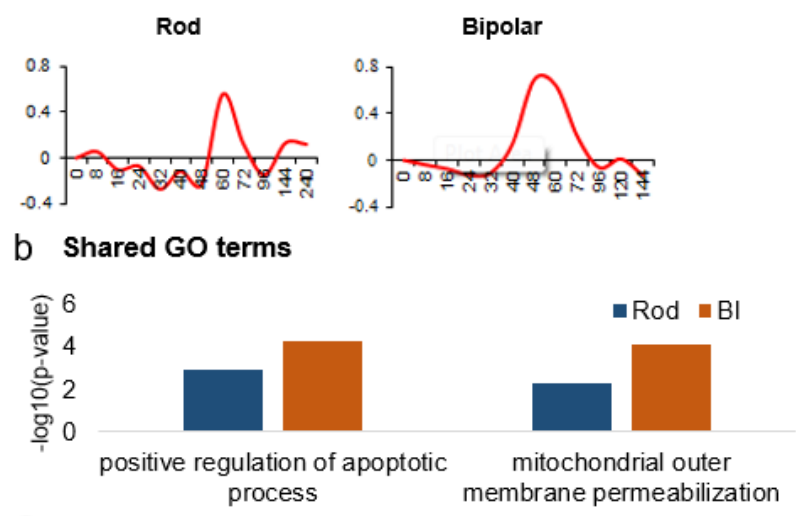

C Paradigm-specific GO terms

transcription, DNA-templated

regulation of transcription, DNA-templated

paraxial mesoderm development

steroid hormone mediated signaling pathway

autophagosome assembly

autophagy

mitophagy

protein localization to pre-autophagosomal structure

activation of MAPKKK activity

protein lipidation

$-\log 10(p-v a l u e)$
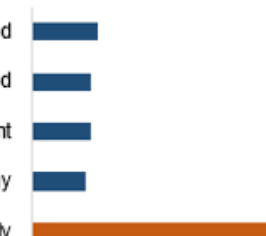

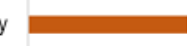
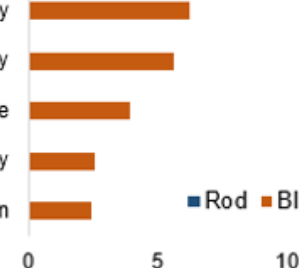

d In-common genes per GO term

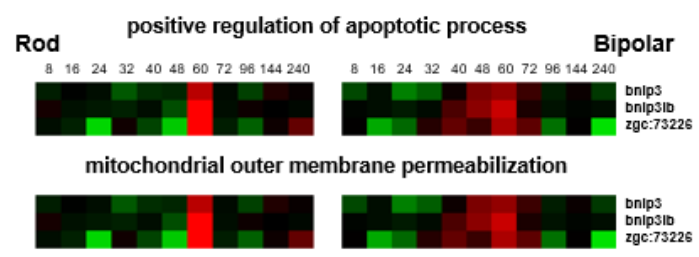

e

Kegg Pathway Analysis

\begin{tabular}{|c|c|c|c|c|}
\hline \multirow{3}{*}{ Rod } & & Function & Gene \# & $p$-value \\
\hline & 1 & Phototransduction & 3 & $7.7 E-2$ \\
\hline & 2 & $A B C$ transporters & 3 & $8.4 E-2$ \\
\hline \multirow{5}{*}{ Bipolar } & 1 & FoxO signaling pathway & 10 & $3.4 E-4$ \\
\hline & 2 & Herpes simplex infection & 9 & $2.3 E-3$ \\
\hline & 3 & Toll-like receptor signaling pathway & 6 & $9.3 E-3$ \\
\hline & 4 & p53 signaling pathway & 5 & $1.4 E-2$ \\
\hline & 5 & Jak-STAT signaling pathway & 5 & $5.6 E-2$ \\
\hline ed & 1 & Lysosome & $7 / 10$ & $1.3 E-2 / 9.6 E-5$ \\
\hline
\end{tabular}

Figure 6: Analysis of Pattern 3; genes upregulated in late regeneration in Rod and Bipolar paradigms. Graphs corresponding to relative expression over the full experiment duration of genes upregulated late (2-fold difference compared to control) in regeneration in both paradigms (a). Gene ontology (GO) was used to identify shared pathway regulatory changes between Rod PRs (Rod) and Bipolar (BI) cells (b) and then paradigm-specific changes (c), listed terms reached shared significance $(\mathrm{p}<0.05)$. Then, GO was further broken down into commonly differentially regulated genes and associated GO terms (d). Lastly, KEGG pathway analysis was used to find paradigm-specific and shared pathway changes that reached significance $(\mathrm{p}<0.05)(\mathrm{e})$. 


\section{Figure 7. Genes downregulated late in regeneration}

a

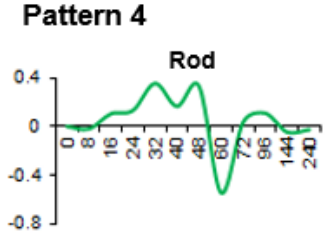

b Shared GO terms

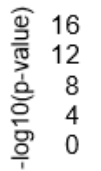

C

Paradigm-specific GO terms

tRNA aminoacylation for protein translation response to light stimulus circadian rhythm response to yeast cell redox homeostasis nuclear mRNA surveillance ribosome biogenesis tetrapyrrole biosynthetic process heme biosynthetic process

$$
\begin{array}{r}
\text { translation } \\
\text { cell cycle } \\
\text { cell division }
\end{array}
$$

retina development in camera-type eye cellular response to DNA damage stimulus
microtubule-based process

mitotic chromosome condensation

$$
-\log 10 \text { (p-value) }
$$

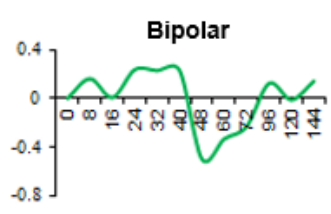

d
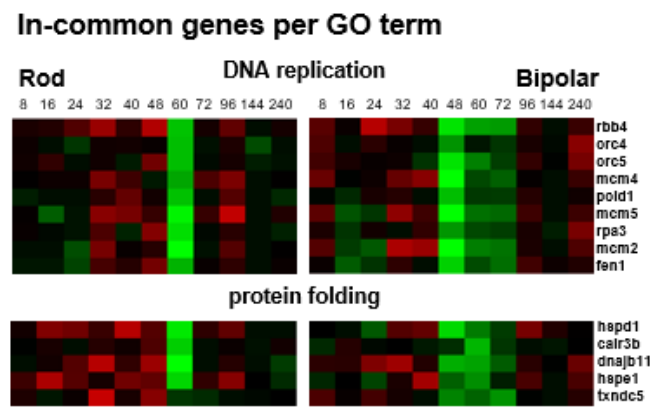

e

Kegg Pathway Analysis

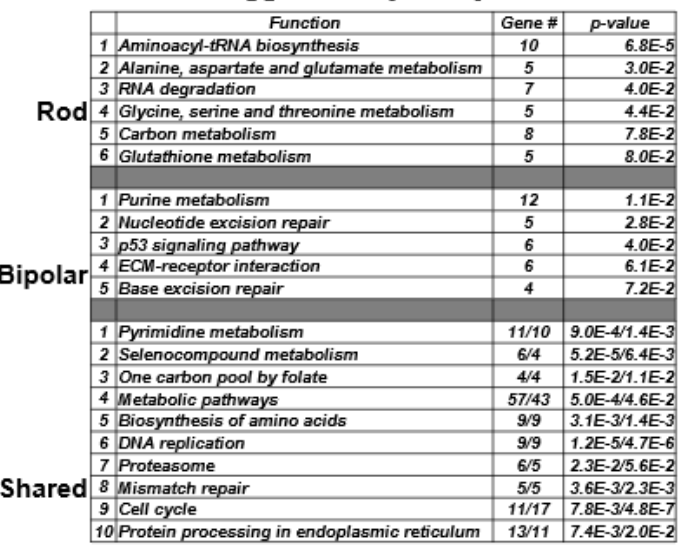

Figure 7: Analysis of Pattern 4; genes downregulated in late regeneration in Rod and Bipolar paradigms. Graphs corresponding to relative expression over the full experiment duration of genes downregulated late (2-fold difference compared to control) in regeneration in both paradigms (a). Gene ontology (GO) was used to identify shared pathway regulatory changes between Rod PRs (Rod) and Bipolar (BI) cells (b) and then paradigm-specific changes (c), listed terms reached shared significance $(\mathrm{p}<0.05)$. Then, GO was further broken down into commonly differentially regulated genes and associated GO terms (d). Lastly, KEGG pathway analysis was used to find paradigm-specific and shared pathway changes that reached significance $(\mathrm{p}<0.05)(\mathrm{e})$. 